\title{
The Hidden Robot: an Efficient Concept Contributing to the Analysis of the Controllability of Parallel Robots in Advanced Visual Servoing Techniques
}

\author{
Sébastien Briot, Philippe Martinet, and Victor Rosenzveig
}

\begin{abstract}
Previous works on parallel robots have shown that their visual servoing using the observation of their leg directions was possible. There were however found two main results for which no answer was given. These results were that (i) the observed robot which is composed of $n$ legs could be controlled in most cases using the observation of only $m$ leg directions $(m<n)$, and that (ii) in some cases, the robot did not converge to the desired end-effector pose, even if the observed leg directions did (i.e. there was not a global diffeomorphism between the observation space and the robot space).

Recently, it was shown that the visual servoing of the leg directions of the Gough-Stewart platform and the Adept Quattro was equivalent to controlling other virtual robots that have assembly modes and singular configurations different from those of the real ones. These hidden robot models are tangible visualizations of the mapping between the observation space and the real robots Cartesian space. Thanks to this concept, all the aforementioned points pertaining to the studied robots were answered.

In this paper, the concept of the hidden robot model is generalized for any type of parallel robots controlled using visual servos based on the observation of elements other than the endeffector, such as the robot legs into motion. It is shown that the concept of the hidden robot model is a powerful tool that gives useful insights about the visual servoing of robots and that it helps define the necessary features to observe in order to ensure the controllability of the robot in its whole workspace. All theoretical concepts are validated through simulations with an Adams mockup linked to Simulink.
\end{abstract}

Index Terms-Parallel robots, visual servoing, controllability, kinematics, singularity.

\section{INTRODUCTION}

Many research papers focus on the control of parallel mechanisms (see [1] for a long list of references). Cartesian control is naturally achieved through the use of the inverse differential kinematic model which transforms Cartesian velocities into joint velocities. It is noticeable that, in a general manner, the inverse differential kinematic model of parallel mechanisms does not only depend on the joint configuration (as for serial mechanisms) but also on the end-effector pose. Consequently, one needs to be able to estimate or measure the latter.

S. Briot, P. Martinet and V. Rosenzveig are with the Institut de Recherche en Communications et Cybernétique de Nantes (IRCCyN), UMR CNRS 6597, Nantes, France, Emails:

\{Sebastien.Briot,Philippe.Martinet,Victor.Rosenzveig\}@irccyn.ec-nantes.fr

P. Martinet is also with the École Centrale de Nantes, France

Manuscript received ...
Past research works proved that the robot end-effector pose can be effectively estimated by vision through the direct [2][4], or the indirect observation of the end-effector pose [5][7]. Visual servoing of parallel robots first focused on the observation of the end-effector [8]-[11]. However, some applications prevent the observation of the end-effector of a parallel mechanism by vision. For instance, it is not wise to imagine observing the end-effector of a machine-tool while it is generally not a problem to observe its legs that are most often designed with slim and rectilinear rods [1].

A first step in this direction was made in [12] where vision was used to derive a visual servoing scheme based on the observation of a Gough-Stewart (GS) parallel robot [13]. In that method, the leg directions were chosen as visual primitives and control was derived based on their reconstruction from the image. By observing several legs, a control scheme was derived and it was then shown that such an approach allowed the control of the observed robot. After these preliminary works, the approach was extended to the control of the robot directly in the image space through the observation of the leg edges (from which the leg direction could be extracted), which proved to exhibit better performances in terms of accuracy than the previous approach [14]. The approach was applied to several types of robots, such as the Adept Quattro and other robots of the same family [15], [16]. As shown in these papers, in order to rebuild the robot configuration from the leg directions (or edges) observation, simplified kinematic models were used.

The proposed control scheme was not usual in visual servoing techniques [17], in the sense that in the controller, both robot kinematics and observation models linking the Cartesian space to the leg direction space were involved. As a result, some surprising results were obtained:

1) the observed robot which is composed of $n$ legs could be controlled in most cases using the observation of only $m$ leg directions $(m<n)$, knowing the fact that the minimal number of observed legs should be, for 3D unit vectors, an integer greater than $n / 2$,

2) in some cases, the robot did not converge to the desired end-effector pose (even if the observed leg directions did)

without finding some concrete explanations to these points.

In parallel, some important questions were never answered, such as: 
3) Are we sure that there is no singularity in the mapping between the leg direction space and the Cartesian space?

4) How can we be sure that the stacking of the observation matrices cannot lead to local minima in the Cartesian space (for which the error in the observation space is non zero while the robot platform cannot move [18])?

All these points were never answered because of the lack of existing tools able to analyze the intrinsic properties of the controller.

Recently, two of the authors of the present paper demonstrated in [19] that these points could be explained by considering that the visual servoing of the leg direction of the GS platform was equivalent to controlling another robot "hidden" within the controller, the $3-\underline{U P S} S^{1}$ that has assembly modes and singular configurations different from those of the GS platform. A similar property was shown for the control of the Adept Quattro for which another hidden robot model, completely different from the one of the GS platform, was found [21]. All theoretical results were validated through experimental works in [22].

In both cases, considering this hidden robot model allowed a minimal representation to be found for the leg-observationbased control of the studied robots that is linked to a virtual hidden robot which is a tangible visualization of the mapping between the observation space and the real robot Cartesian space.

Thus, the concept of the hidden robot model, associated with mathematical tools developed by the mechanical design community, is a powerful tool able to analyze the intrinsic properties of some controllers developed by the visual servoing community. Moreover, this concept shows that in some visual servoing approaches, stacking several interaction matrices to derive a control scheme without doing a deep analysis of the intrinsic properties of the controller is clearly not enough. Further investigations are required.

Therefore, in this paper, the generalization of the concept of hidden robot model is presented and a general way to find the hidden robots corresponding to any kind of robot architecture is explained. It will be shown that the concept of the hidden robot model is a powerful tool that gives useful insights about the visual servoing of robots using leg direction observation. With the concept of the hidden robot model, the singularity problem of the mapping between the space of the observed robot links and the Cartesian space can be addressed, and above all, it is possible to give and certify information about the controllability of the observed robots using the proposed controller.

Some parts of the present works were published in [22]. However, the present paper presents for the first time:

- a classification into families of robots which are not controllable, partially or fully controllable in their whole workspace using the aforementioned servoing technique,

- insights about the features that should be additionally observed to ensure that the robots could be fully controllable in their whole workspace.

${ }^{1}$ In the following of the paper, $R, P, U, S, \Pi$ will stand for passive revolute, prismatic, universal, spherical and planar parallelogram joint [20], respectively. If the letter is underlined, the joint is considered active.
Finally, we would like to mention that, in the present paper, we will define the concept of the hidden robot model based on the 3D primitives (leg directions) used in the controller defined in [12], even if the results provided in [14] by using the observation of the leg edges proved to exhibit better performances in terms of accuracy than the previous approach. However, deriving the hidden robot model using the leg edges would lead to more complex and much longer explanations. Nevertheless, the results shown in the present paper are generic enough to be then applied to other types of controllers, such as the one given in [14].

\section{VISUAL SERVOING OF PARALLEL ROBOTS USING LEG OBSERVATIONS}

\section{A. Line modeling}

A line $\mathcal{L}$ in space, expressed in the camera frame, is defined by its Binormalized Plücker coordinates [23]:

$$
\mathcal{L} \equiv\left({ }^{c} \underline{\mathbf{u}},{ }^{c} \underline{\mathbf{n}},{ }^{c} n\right)
$$

where ${ }^{c} \underline{\mathbf{u}}$ is the unit vector giving the spatial orientation of the line $^{2},{ }^{c} \underline{\mathbf{n}}$ is the unit vector defining the so-called interpretation plane of line $\mathcal{L}$ and ${ }^{c} n$ is a non-negative scalar. The latter are defined by ${ }^{c} n^{c} \underline{\mathbf{n}}={ }^{c} \mathbf{p} \times{ }^{c} \underline{\mathbf{u}}$ where ${ }^{c} \mathbf{p}$ is the position of any point $P$ on the line, expressed in the camera frame. Notice that, using this notation, the well-known (normalized) Plücker coordinates [24], [25] are the couple $\left({ }^{c} \underline{\mathbf{u}},{ }^{c} n^{c} \underline{\mathbf{n}}\right)$.

The projection of such a line in the image plane, expressed in the camera frame, has the characteristic equation [23]:

$$
{ }^{c} \underline{\mathbf{n}}^{T c} \mathbf{p}=0
$$

where ${ }^{c} \mathbf{p}$ are the coordinates in the camera frame of a point $P$ in the image plane, lying on the line.

\section{B. Cylindrical leg observation}

The legs of parallel robots usually have cylindrical crosssections [25]. The edges of the $i$-th cylindrical leg are given, in the camera frame, by [14] (Fig 1):

$$
\begin{aligned}
& { }^{c} \underline{\mathbf{n}}_{i}^{1}=-\cos \theta_{i}{ }^{c} \underline{\mathbf{h}}_{i}-\sin \theta_{i}{ }^{c} \underline{\mathbf{u}}_{i} \times{ }^{c} \underline{\mathbf{h}}_{i} \\
& { }^{c} \underline{\mathbf{n}}_{i}^{2}=+\cos \theta_{i}{ }^{c} \underline{\mathbf{h}}_{i}-\sin \theta_{i}{ }^{c} \underline{\mathbf{u}}_{i} \times{ }^{c} \underline{\mathbf{h}}_{i}
\end{aligned}
$$

where $\cos \theta_{i}=\sqrt{{ }^{c} h_{i}^{2}-R_{i}^{2}} /{ }^{c} h_{i}, \sin \theta_{i}=R_{i} /{ }^{c} h_{i}$ and $\left({ }^{c} \underline{\mathbf{u}}_{i}, \underline{\mathbf{h}}_{i},{ }^{c} h_{i}\right)$ are the Binormalized Plücker coordinates of the cylinder axis and $R_{i}$ is the cylinder radius.

It was also shown in [14] that the leg orientation, expressed in the camera frame, is given by

$$
{ }^{c} \underline{\mathbf{u}}_{i}=\frac{{ }^{c} \underline{\mathbf{n}}_{i}^{1} \times{ }^{c} \underline{\mathbf{n}}_{i}^{2}}{\left\|\underline{\mathbf{n}}_{i}^{1} \times{ }^{c} \underline{\mathbf{n}}_{i}^{2}\right\|}
$$

Let us remark that each cylinder edge is a line in space, with Binormalized Plücker expressed in the camera frame $\left({ }^{c} \underline{\mathbf{u}}_{i},{ }^{c} \underline{\mathbf{n}}_{i}^{j},{ }^{c} n_{i}^{j}\right)$ (Fig 1).

\footnotetext{
${ }^{2}$ In the following of the paper, the superscript before the vector denotes the frame in which the vector is expressed (" $b$ " for the base frame, " $c$ " for the camera frame and " $p$ " for the pixel frame). If there is no superscript, the vector can be written in any frame.
} 


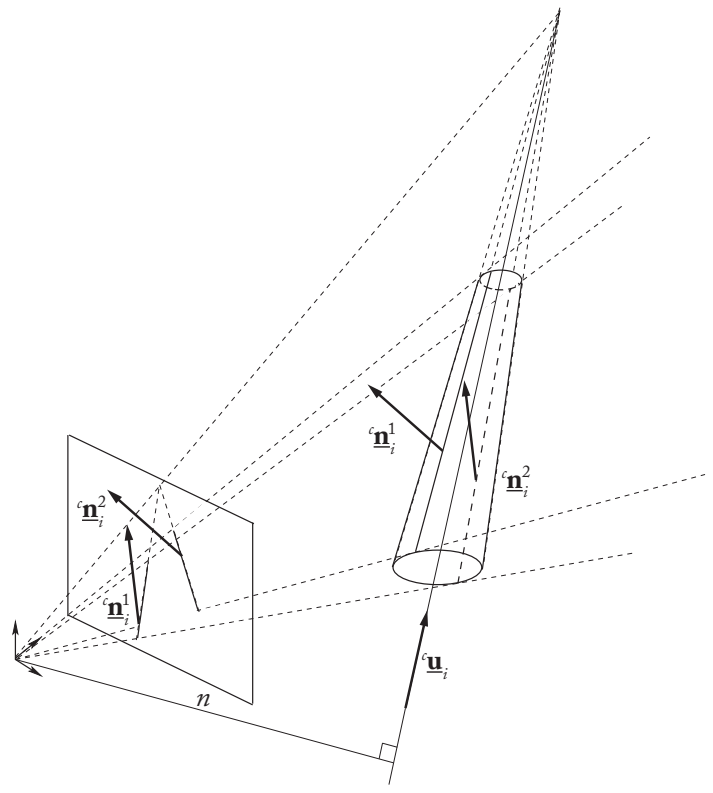

Fig. 1. Projection of a cylinder in the image

\section{Leg direction based visual servoing}

The proposed control approach was to servo the leg directions ${ }^{c} \underline{\mathbf{u}}_{i}$ [12]. Some brief recalls on this type of controller are done below.

1) Interaction matrix: Visual servoing is based on the socalled interaction matrix $\mathbf{L}^{T}$ [26] which relates the instantaneous relative motion $T_{c}={ }^{c} \boldsymbol{\tau}_{c}-{ }^{c} \boldsymbol{\tau}_{s}$ between the camera and the scene, to the time derivative of the vector $s$ of all the visual primitives that are used through:

$$
\dot{s}=\mathbf{L}_{(s)}^{T} T_{c}
$$

where ${ }^{c} \boldsymbol{\tau}_{c}$ and ${ }^{c} \boldsymbol{\tau}_{s}$ are respectively the kinematic screw of the camera and the scene, both expressed in $\mathcal{R}_{c}$, i.e. the camera frame.

In the case where we want to directly control the leg directions ${ }^{c} \underline{\mathbf{u}}_{i}$, and if the camera is fixed, (6) becomes:

$$
{ }^{c} \underline{\dot{\mathbf{u}}}_{i}=\mathbf{M}_{i}^{T c} \boldsymbol{\tau}_{c}
$$

where $\mathbf{M}_{i}^{T}$ is the interaction matrix for the leg $i$.

2) Control: For the visual servoing of a robot, one achieves exponential decay of an error $e\left(s, s_{d}\right)$ between the current primitive vector $s$ and the desired one $s_{d}$ using a proportional linearizing and decoupling control scheme of the form (if the scene is fixed):

$$
{ }^{c} \boldsymbol{\tau}_{c}=\lambda \hat{\mathbf{L}}_{(s)}^{T+} e\left(s, s_{d}\right)
$$

where ${ }^{c} \boldsymbol{\tau}_{c}$ is used as a pseudo-control variable and the superscript "+" corresponds to the matrix pseudo-inverse.

The visual primitives being unit vectors, it is theoretically more elegant to use the geodesic error rather than the standard vector difference. Consequently, the error grounding the proposed control law will be:

$$
\mathbf{e}_{i}={ }^{c} \underline{\mathbf{u}}_{i} \times{ }^{c} \underline{\mathbf{u}}_{d i}
$$

where ${ }^{c} \underline{\mathbf{u}}_{d i}$ is the desired value of ${ }^{c} \underline{\mathbf{u}}_{i}$.
It can be proven that, for spatial parallel robots, matrices $\mathbf{M}_{i}$ are in general of rank 2 [12] (for planar parallel robots, they are of rank 1). As a result, for spatial robots with more than 2 degrees of freedom (dof), the observation of several independent legs is necessary to control the end-effector pose. An interaction matrix $\mathbf{M}^{T}$ can then obtained by stacking $k$ matrices $\mathbf{M}_{i}^{T}$ of $k$ legs.

Finally, a control is chosen such that e, the vector stacking the errors $\mathbf{e}_{i}$ of $k$ legs, decreases exponentially, i.e. such that

$$
\dot{\mathbf{e}}=-\lambda \mathbf{e}
$$

It should be mentioned that, in reality, it is not possible to ensure a perfect exponential decrease of $\mathbf{e}$ if the dimension of $\mathbf{e}$ is larger than the number of degrees of freedom [27], [28].

Then, introducing $\mathbf{L}_{i}^{T}=-\left[{ }^{c} \underline{\mathbf{u}}_{d i}\right]_{\times} \mathbf{M}_{i}^{T}$, where $\left[{ }^{c} \underline{\mathbf{u}}_{d i}\right]_{\times}$is the cross product matrix associated with the vector ${ }^{c} \underline{\mathbf{u}}_{d i}$, the combination of (9), (7) and (10) gives

$$
{ }^{c} \boldsymbol{\tau}_{c}=-\lambda \mathbf{L}^{T+} \mathbf{e}
$$

where $\mathbf{L}^{T}$ can be obtained by stacking the matrices $\mathbf{L}_{i}^{T}$ of $k$ legs. The conditions for the rank deficiency of matrix $\mathbf{L}^{T}$, as well as the conditions that lead to local minima [18] of the Eq. (11) are discussed in Section III.

This expression can be transformed into the control joint velocities:

$$
\dot{\mathbf{q}}=-\lambda^{c} \mathbf{J}^{i n v} \mathbf{L}^{T+} \mathbf{e}
$$

where ${ }^{c} \mathbf{J}^{i n v}$ is the inverse Jacobian matrix of the robot relating the end-effector twist to the actuator velocities, i.e. ${ }^{c} \mathbf{J}^{i n v c} \boldsymbol{\tau}_{c}=$ $\dot{\mathbf{q}}$.

\section{Statement of the problem}

It is obvious that the objective of any controller is to ensure two main properties: the observability of some given robot elements (in our case, the end-effector) and the controllability of the robot. For that, any controller is based on the observation of some features (the encoder positions, velocity and acceleration in usual controllers, or some robot parts in sensorbased controllers) which must ensure that:

1) it is possible to properly estimate the pose (and also eventually the velocity and acceleration) of the endeffector (which is an external property of the robot),

2) it is also possible to estimate the internal state of the robot (position, velocity and acceleration of any body) as this information is necessary for achieving the control (for instance, in the controller defined at Eq. (12), the computation of the inverse kinematic Jacobian matrix ${ }^{c} \mathbf{J}^{i n v}$ is necessary, and its expression is usually a function of the active (and sometimes also passive) joint variables).

Ideally, from the observation of a minimal set of given features (denoted as a minimal basis), the mapping involved for the estimation of the end-effector pose must be a global diffeomorphism (Fig. 2(a)). However, in the case of parallel robots in classical encoder-based controllers, a given set of encoder positions usually leads to the computation of several possible end-effector poses [25] which are called the robot 


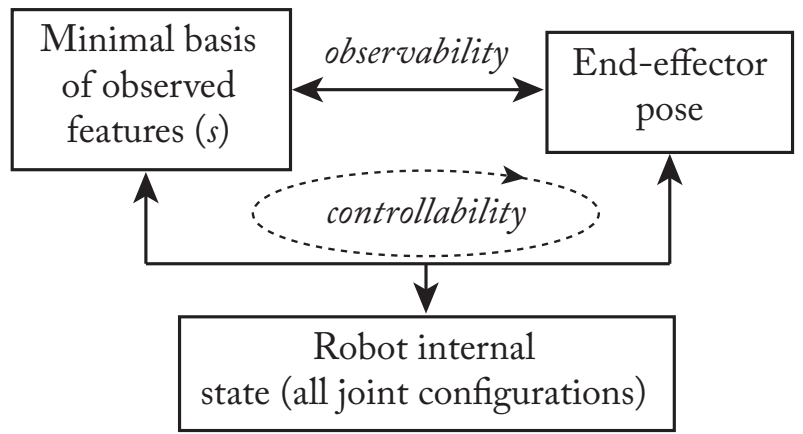

(a) when a global diffeomorphism exists

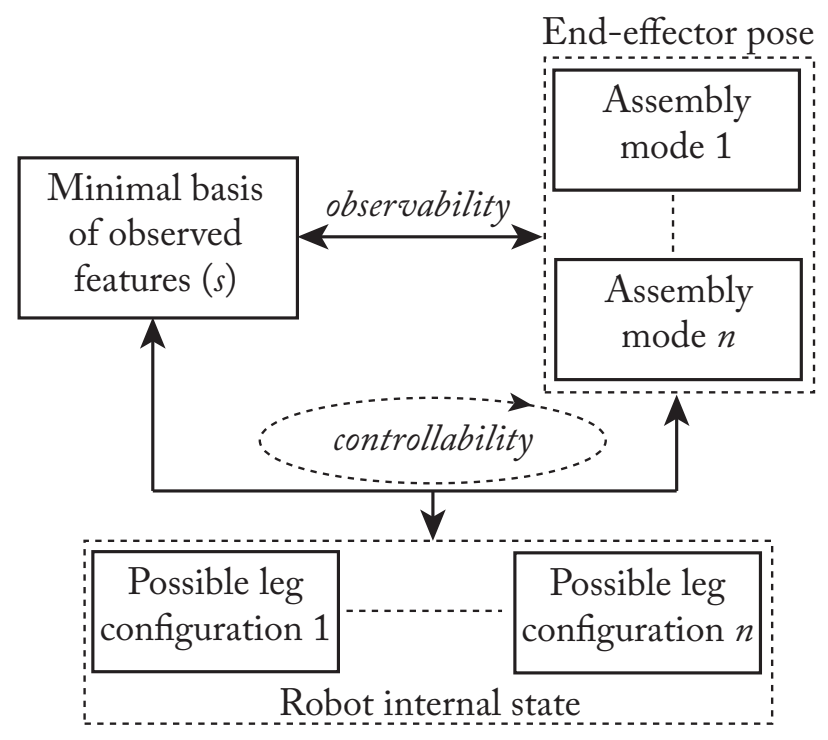

(b) when there is no global diffeomorphism

Fig. 2. Ensuring the observability and controllability of the robot through a proper feature observation.

assembly modes. These assembly modes correspond to some given aspects of the workspace (i.e. workspace zones which are seperated by singularities), which means that the robot cannot freely move in all the workspace areas. Thus, there is no global diffeomorphism between the encoder positions and the end-effector pose (Fig. 2(b)). To overcome this difficulty, usually, the parallel robot is moved in only one given workspace aspect for which the assembly mode can be strictly known.

By extension, if we cannot strictly know the end-effector pose, we cannot also correctly estimate the internal robot state (position, velocity and acceleration of any body) ${ }^{3}$. The question is thus: what should be the minimal basis of the observed features that is able to ensure that we are able to stricly estimate both the end-effector pose and the robot

\footnotetext{
${ }^{3}$ It is necessary to mention that, for a given end-effector pose, several leg configurations (called working modes) may exist. However, for the large majority of parallel robots for which each leg is made of at most two moving elements, if we strictly know the end-effector pose plus the pose of an element of a considered leg, the leg configuration can be uniquely defined.
}

internal state, i.e. to strictly ensure the robot controllability?

In the next Sections, it is shown that the use of a tool named the "hidden robot model" can help analyze the controllability of parallel robots when the canonical basis of the observed features is partially made of the robot leg directions. We first introduce the concept of the hidden robot model and then show how it can be used for the analysis of the controllability.

\section{THE CONCEPT OF HIDDEN ROBOT MODEL}

The concept of the hidden robot model was first introduced in [19] for the visual servoing of the GS platform. In this paper, it has been demonstrated that the leg-direction-based visual servoing (Section II) of such robots intrinsically involves the appearance of a hidden robot model, which has assembly modes and singularities different from the real robot. It was shown that the concept of the hidden robot model fully explains the possible non-convergence of the observed robot to the desired final pose and that it considerably simplifies the singularity analysis of the mapping involved in the controller.

The concept of the hidden robot model comes from the following observation: in the classical control approach, the encoders measure the motion of the actuator; in the previously described control approach (Section II), the leg directions or leg edges are observed. So, in a reciprocal manner, one could wonder to what kind of virtual actuators such observations correspond. The main objective of this Section is to give a general answer to this question.

\section{A. How to define the legs of the hidden robots}

Let us consider a general leg for a parallel robot in which the direction $\underline{\mathbf{u}}_{i}$ of a segment is observed (Fig. 3(a) - in this figure, the last segment is considered observed, but the following explanations can be generalized to any segment located in the leg chain). In what follows, we only consider that we observe the leg direction $\underline{\mathbf{u}}_{i}$, and not the leg edges in the image space, as the leg edges are only used as a measure of $\underline{\mathbf{u}}_{i}$. So the problem is the same, except in the fact that we must consider the singularity of the mapping between the edges and $\underline{\mathbf{u}}_{i}$, but this problem is well handled: these singularities appear when $\underline{\mathbf{n}}_{i}^{1}$ and $\underline{\mathbf{n}}_{i}^{2}$ are collinear, i.e. the cylinders are at infinity [14].

In the general case, the unit vector $\underline{\mathbf{u}}_{i}$ can obviously be parameterized by two independent coordinates, that can be two angles, for example the angles $\alpha$ and $\beta$ of Fig. 4 defined such that $\cos \alpha=\underline{\mathbf{x}} \cdot \underline{\mathbf{v}}=\underline{\mathbf{y}} \cdot \underline{\mathbf{w}}$ (where $\underline{\mathbf{v}}$ and $\underline{\mathbf{w}}$ are defined such that $\underline{\mathbf{z}} \cdot \underline{\mathbf{v}}=\underline{\mathbf{z}} \cdot \underline{\mathbf{w}}=0$ ) and $\cos \beta=\underline{\mathbf{u}} \cdot \underline{\mathbf{x}}$. Thus $\alpha$ is the angle of the first rotation of the link direction $\underline{\mathbf{u}}_{i}$ around $\underline{\mathbf{z}}$ and $\beta$ is the angle of the second rotation around $\underline{\mathbf{v}}$.

It is well known that a $U$ joint is able to orient a link around two orthogonal axes of rotation, such as $\underline{\mathbf{z}}$ and $\underline{\mathbf{v}}$. Thus $U$ joints can be the virtual actuators we are looking for, with generalized coordinates $\alpha$ and $\beta$. Of course, other solutions can exist, but $U$ joints are the simplest ones.

If a $U$ joint is the virtual actuator that makes the vector $\underline{\mathbf{u}}_{i}$ move, it is obvious that:

- if the value of $\underline{\mathbf{u}}_{i}$ is fixed, the $U$ joint coordinates $\alpha$ and $\beta$ must be constant, i.e. the actuator must be blocked, 


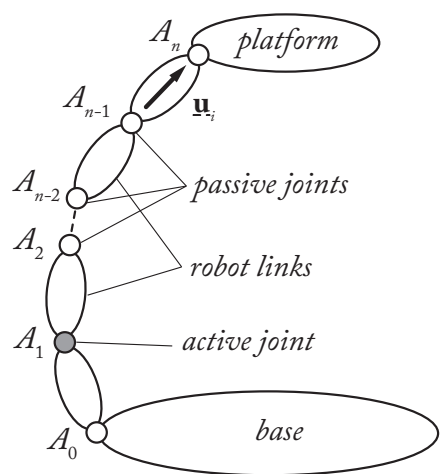

(a) A general robot leg

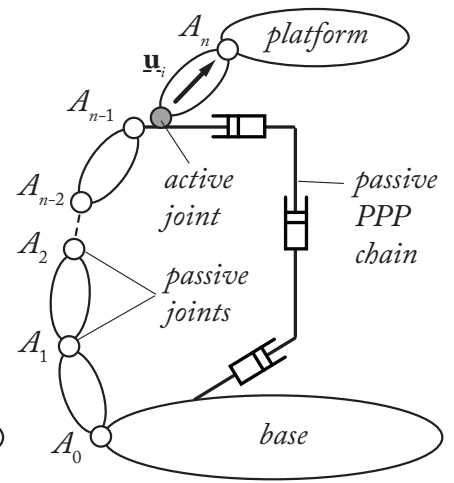

(b) its corresponding hidden robot leg when the vector $\underline{\mathbf{u}}_{i}$ is observed
Fig. 3. A general robot leg and its corresponding hidden robot leg when the vector $\underline{\mathbf{u}}_{i}$ is observed

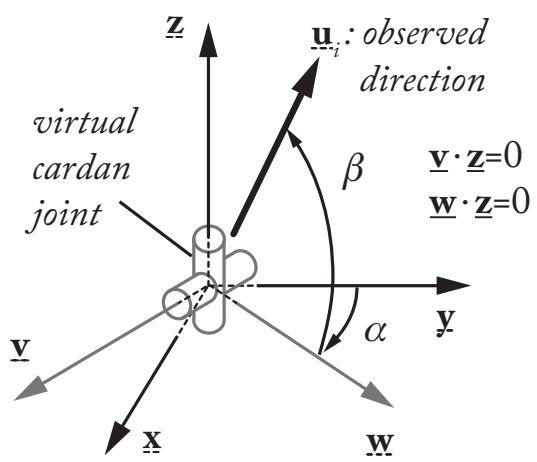

Fig. 4. Parameterization of a unit vector $\underline{\mathbf{u}}_{i}$ with respect to a given frame $\underline{\mathbf{x}}$, $\underline{\mathbf{y}}$ and $\underline{\mathbf{z}}$

- if the value of $\underline{\mathbf{u}}_{i}$ is changing, the $U$ joint coordinates $\alpha$ and $\beta$ must also vary.

As a result, to ensure the aforementioned properties for $\alpha$ and $\beta$ if $\underline{\mathbf{u}}_{i}$ is expressed in the base or camera frame (but the problem is identical as the camera is considered fixed on the ground), vectors $\underline{\mathbf{x}}, \underline{\mathbf{y}}$ and $\underline{\mathbf{z}}$ of Fig. 4 must be the vectors defining the base or camera frame. Thus, in terms of properties for the virtual actuator, this implies that the first $\underline{U}$ joint axis must be constant w.r.t. the base frame, i.e. the $\underline{U}$ joint must be attached to a link performing a translation w.r.t. the base frame ${ }^{4}$.

However, in most cases, the real leg architecture is not composed of $U$ joints attached to links performing a translation w.r.t. the base frame. Thus, the architecture of the hidden robot leg must be modified w.r.t. the real leg such as depicted in Fig. 3(b). The $\underline{U}$ joint must be mounted on a passive kinematic chain composed of at most 3 orthogonal passive $P$ joints that ensures that the link to which it is attached performs a translation w.r.t. the base frame. This passive chain is also linked to the segments before the observed links so that they do not change their kinematic properties in terms of motion. Note that:

\footnotetext{
${ }^{4}$ In the case where the camera is not mounted on the frame but on a moving link, the virtual $\underline{U}$ joint must be attached on a link performing a translation w.r.t. the considered moving link.
}

- it is necessary to fix the $P P P$ chain on the preceding leg links because the information given by the vectors $\underline{\mathbf{u}}_{i}$ is not enough to rebuild the full platform position and orientation: it is also necessary to get information (obtained via simplified kinematic models [14]) on the location of the anchor point $A_{n-1}$ of the observed segment. This information is kept through the use of the PPP chain fixed on the first segments;

- $3 P$ joints are only necessary if and only if the point $A_{n-1}$ describes a motion in the $3 \mathrm{D}$ space; if not, the number of $P$ joints can be decreased: for example, in the case of the GS platform presented in [19], the $U$ joint of the leg to control was located on the base, i.e. there was no need to add passive $P$ joints to keep the orientation of its first axis constant;

- when the vector $\underline{\mathbf{u}}_{i}$ is constrained to move in a plane such as for planar legs, the virtual actuator becomes an $R$ joint which must be mounted on the passive PPP chain (for the same reasons as mentioned previously).

For example, let us have a look at the $\underline{R} U$ leg with one actuated $\underline{R}$ joint followed by a $U$ joint of Fig. 5(a). Using the previous approach, its virtual equivalent leg should be an $\{R-$ $P P\}-\underline{U}$ leg (Fig. 5(b)), i.e. the $\underline{U}$ joint able to orient the vector $\underline{\mathbf{u}}_{i}$ is mounted on the top of a $R-P P$ chain that can guarantee that:

1) the link on which the $\underline{U}$ joint is attached performs a translation w.r.t. the base frame,

2) the point $C$ (i.e. the centre of the $U$ joint) evolves on a circle of radius $l_{A B}$, like the real leg.

It should be noted that, in several cases for robots with a lower mobility (i.e. spatial robots with a number of dof less than 6 , or planar robots with a number of dof less than 3 ), the last joint that links the leg to the platform should be changed so that, if the number of observed legs is inferior to the number of real legs, the hidden robot keeps the same number of controlled dof (see [21], [22]).

It should also be mentioned that we presented above the most general methodology that is possible to propose, but it is not the most elegant way to proceed. In many cases, a hidden robot leg architecture can be obtained such that less modifications w.r.t the real leg are achieved. For example, the $R-P P$ chain of the hidden robot leg $\{R-P P\}-\underline{U}$ (Fig. 5(b)) could be equivalently replaced by a planar parallelogram (П) joint without changing the aforementioned properties of the $\underline{U}$ virtual actuator (Fig. 5(c)), i.e. only one additional joint is added to obtain the hidden robot leg (note that we consider that a $\Pi$ joint, even if composed of several pairs, can be seen as one single joint, as in [20]).

In what follows in this paper, this strategy for finding the simplest hidden robot legs (in terms of architectural simplicity) is adopted for the studied robots.

B. How to use the hidden robot models for understanding the surprising and unanswered results arising from the use of legdirection-based controllers

The aim of this Section is to show how to use the hidden robots to answer points 1 to 4 enumerated in the introduction 


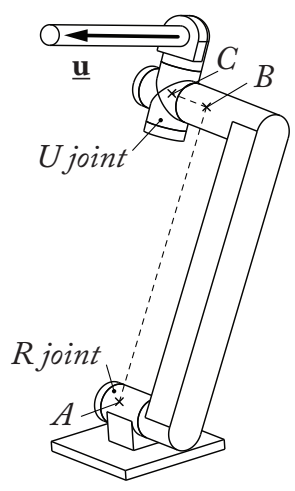

(a) A $\underline{R} U$ leg

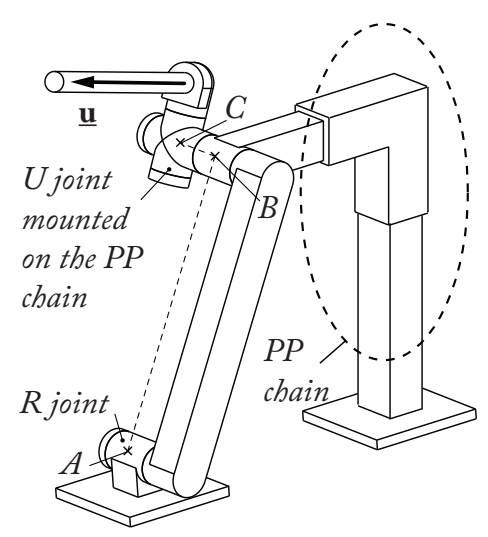

(b) Virtual $\{R-P P\}-\underline{U}$ leg

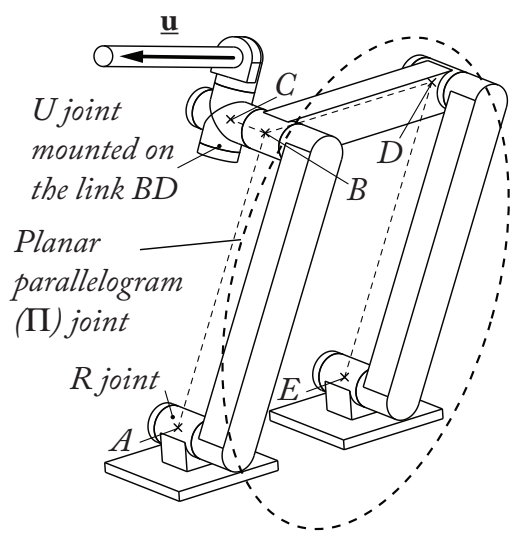

(c) Virtual $\Pi \underline{U}$ leg

Fig. 5. A $\underline{R} U$ leg and two equivalent solutions for its hidden leg

of the paper.

Point 1: the hidden robot model can be used to explain why the observed robot which is composed of n legs can be controlled using the observation of only $m$ leg directions $(m<n)$.

To answer this point, let us consider a general parallel robot composed of 6 legs (one actuator per leg) and having six $d o f$. Using the approach proposed in Section III-A, each

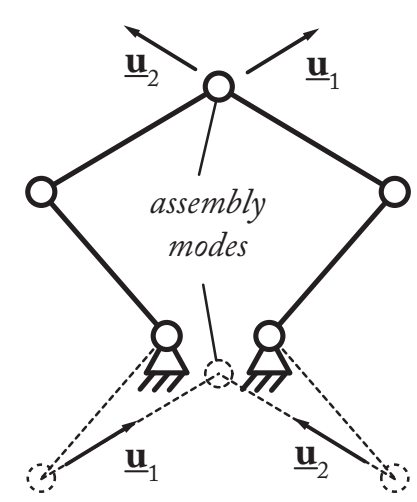

Fig. 6. Two configurations of a five bar mechanism for which the directions $\underline{\mathbf{u}}_{i}$ are identical (for $i=1,2$ )

observed leg will lead to a modified virtual leg with at least one actuated $\underline{U}$ joint that has two degrees of actuation. For controlling $6 \mathrm{dof}$, only 6 degrees of actuation are necessary, i.e. three actuated $\underline{U}$ are enough (as long as the motions of the $\underline{U}$ joints are not correlated, i.e. the robot is fully actuated). Thus, in a general case, only three legs have to be observed to fully control the platform $d o f$.

Point 2: the hidden robot model can be used to prove that there does not always exist a global diffeomorphism between the Cartesian space and the leg direction space.

Here, the answer comes directly from the fact that the real controlled robot may have a hidden robot model with different geometric and kinematics properties. This means that the hidden robot may have assembly modes and singular configurations different from those of the real robot. If the initial and final robot configurations are not included in the same aspect (i.e. a workspace area that is singularity-free and bounded by singularities [25]), the robot will not be able to converge to the desired pose, but to a pose that corresponds to another assembly mode that has the same leg directions as the desired final pose (see Fig. 6).

Point 3: the hidden robot model simplifies the singularity analysis of the mapping between the leg direction space and the Cartesian space by reducing the problem to the singularity analysis of a new robot.

The interaction matrix $\mathbf{M}^{T}$ involved in the controller gives the value of ${ }^{c} \underline{\underline{\dot{u}}}$ as a function of ${ }^{c} \boldsymbol{\tau}_{c}$. Thus, $\mathbf{M}^{T}$ is the inverse kinematic Jacobian matrix of the hidden robot (and, consequently, $\mathbf{M}^{T+}$ is the hidden robot kinematic Jacobian matrix). Except in the case of decoupled robots [29]-[31], the kinematic Jacobian matrices of parallel robots are not free of singularities.

Considering the input/output relations of a robot, three 
different kinds of singularity can be observed $[32]^{5}$ :

- the Type 1 singularities that appear when the robot kinematic Jacobian matrix is rank-deficient; in such configurations, any motion of the actuator that belongs to the kernel of the kinematic Jacobian matrix is not able to produce a motion of the platform,

- the Type 2 singularities that occur when the robot inverse kinematic Jacobian matrix is rank-deficient; in such configurations, any motion of the platform that belongs to the kernel of the inverse kinematic Jacobian matrix is not able to produce a motion of the actuator. And, reciprocally, near these configurations, small motions of the actuators lead to large platform displacements, i.e. the accuracy of the robot becomes very poor,

- the Type 3 singularities that appear when both the robot kinematic Jacobian and inverse kinematic Jacobian matrices are rank-deficient.

Thus,

- finding the condition for the rank-deficiency of $\mathbf{M}^{T}$ is equivalent to finding the Type 2 singularities of the hidden robot,

- finding the condition for the rank-deficiency of $\mathbf{M}^{T+}$ is equivalent to finding the Type 1 singularities of the hidden robot.

Since a couple of decades ago, many tools have been developed by the mechanical design community for finding the singular configurations of robots. The interested reader could refer to [25], [34]-[36] and many other works on the Grassmann Geometry and Grassmann-Cayley Algebra for studying the singular configurations problem. In what follows in the paper, these tools are used but only the final results concerning the singular configuration conditions are given.

Point 4: the hidden robot model can be used to certify that the robot will not converge to local minima.

The robot could converge to local minima if the matrix $\mathbf{M}^{T+}$ is rank deficient, i.e. the hidden robot model encounters a Type 1 singularity. As mentioned above, many tools have been developed by the mechanical design community for finding the singular configurations of robots and solutions can be provided to ensure that the hidden robot model does not meet any Type 1 singularity.

The next Section explains how to use the hidden robot concept to check the controllability of robots and, eventually for robots which are not controllable, how to modify the controller to ensure their controllability.

\section{Controllability analysis}

Thanks to the hidden robot concept, it is possible to analyze the controllability of parallel robots and to define three categories of robots:

\footnotetext{
${ }^{5}$ There exist other types of singularities, such as the constraint singularities [33], but they are due to passive constraint degeneracy only, and are not involved in the mapping between the leg directions space and the robot controlled Cartesian coordinate space.
}

1) robots which are not controllable using the leg direction observation: this case will appear if, for a given set of observed features $s$, the mapping involved in the controller for estimating the end-effector pose is singular for an infinity of robot configurations (in other words, the end-effector configuration is not observable),

2) robots which are partially controllable in their whole workspace using the leg direction observation: this case will appear if, for a given set of observed features $s$, the mapping involved in the controller is not a global diffeomorphism (i.e. a given set of observed features $s$ may lead to several possible end-effector configurations - Fig. 2(b)),

3) robots which are fully controllable in their whole workspace using the leg direction observation: this case will appear if, for a given set of observed features $s$, the mapping involved in the controller is a global diffeomorphism (i.e. a given set of observed features $s$ leads to a unique end-effector configuration - Fig. 2(a)).

Families of robots belonging to these categories are defined thereafter. Moreover, after this classification, insights are provided to ensure that all robots could be controllable by adding supplementary observations.

\section{A. Robots which are not controllable using the leg direction observation}

With the hidden robot concept, it is possible to find classes of robots which are not controllable using leg observations, and this without any mathematical derivations. These robots are those with a hidden robot model which is architecturally singular (whatever the number of observed legs). In other words, the hidden robots have unconstrained $d o f$.

Three main classes of parallel robots belong to this category (the list is not exhaustive, but groups the most usual and known robots in the community):

- robots with legs whose directions are constant for all robot configurations: for these robots, the anchor point location of the observed links cannot be found through the use of the simplified kinematic models. This are the cases of planar 3-PPR (Fig. 7) and 3-PPR robots [25], [37] and of certain spatial robots such as the $3-[\underline{P P}] P S$ robots $^{6}$ (with 3-PPS robots (with 3 dof [38] (Fig. 8) or with 6 dof - e.g. the MePaM [36])). It is obvious that for robots with legs whose directions are constant in the whole workspace, it is not possible to estimate the platform pose from the leg directions only.

- robots with legs whose directions are constant for an infinity of (but not all) robot configurations: this is the case of $\underline{P} R R R \underline{P}$ robots with all $\underline{P}$ parallel (Fig. 9(a)) and of Delta-like robots actuated via $\underline{P}$ joints for which all $\underline{P}$ are parallel (such as the UraneSX (Fig. 10) or the I4L [39], [40]). It was shown in [16] through the analysis of the rank deficiency of the interaction matrix that it was not possible to control such types of robots using leg direction observation. Considering this problem with

${ }^{6}[\underline{P P}]$ means an active planar chain able to achieve two dof of translation, such as $\underline{P P}$ or $\underline{R R}$ chains 


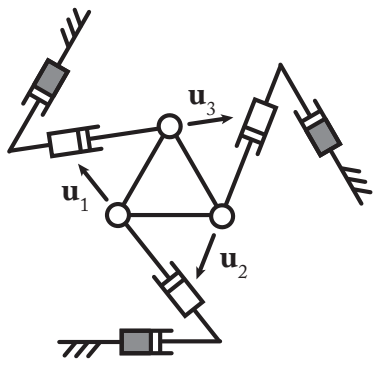

(a) The $3-\underline{P} P R$ robot

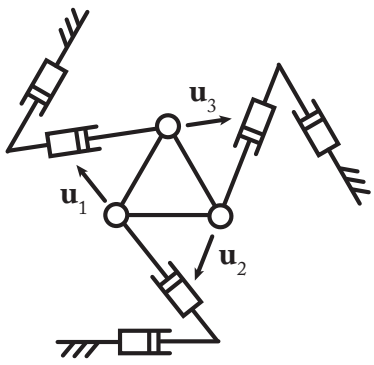

(b) Its hidden robot model: a $3-$ $P P R$ robot with no actuators

Fig. 7. The $3-\underline{P} P R$ robot and its hidden robot model (the grey joints denote the actuated joints)

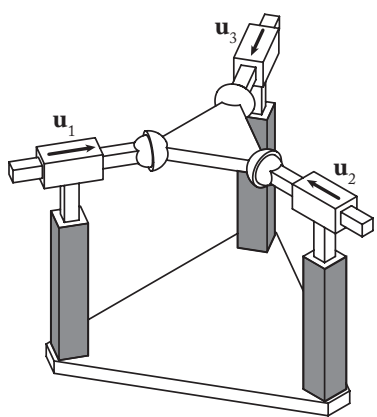

(a) The 3-PPS robot

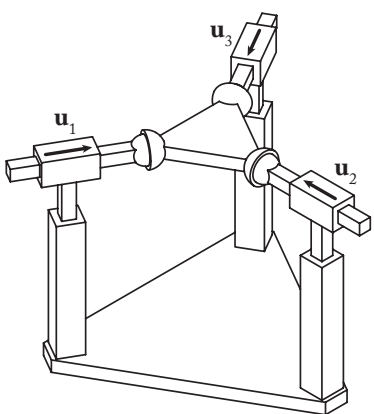

(b) Its hidden robot model: a $3-$ $P P S$ robot with no actuators

Fig. 8. The 3-PPS robot and its hidden robot model (the grey joints denote the actuated joints)

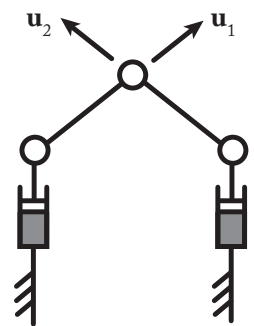

(a) The $\underline{P R R R} \underline{P}$ robot

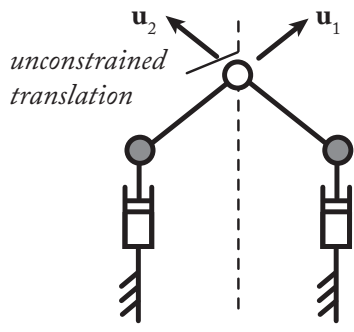

(b) Its hidden robot model: a $P \underline{R R} \underline{R} P$ robot

Fig. 9. The $\underline{P R R R} \underline{P}$ robot and its hidden robot model (the grey circles denote the actuated joints)

the hidden robot concept is very easy. For example, in the case of the $\underline{P R R R} \underline{P}$ robot with parallel $\underline{P}$ joints, the hidden robot has a $P \underline{R} R \underline{R} P$ architecture (Fig. 9(b)), where the parallel $P$ joints are passive. This robot is well-known to be architecturally singular as there is no way to control the translation along the axis of the parallel $P$ joints. This result can be easily extended to the cases of the hidden robots of the UraneSX and the I4L (Fig. 10).

- robots with legs whose directions vary with the robot configurations but for which all hidden robot legs contain active $R$ joints but only passive $P$ joints: the most known robot of this category will be the planar $3-\underline{P} R P$ robot for which the hidden robot model is a $3-P \underline{R} P$ which is known to be uncontrollable [25], [37] (Fig. 11).

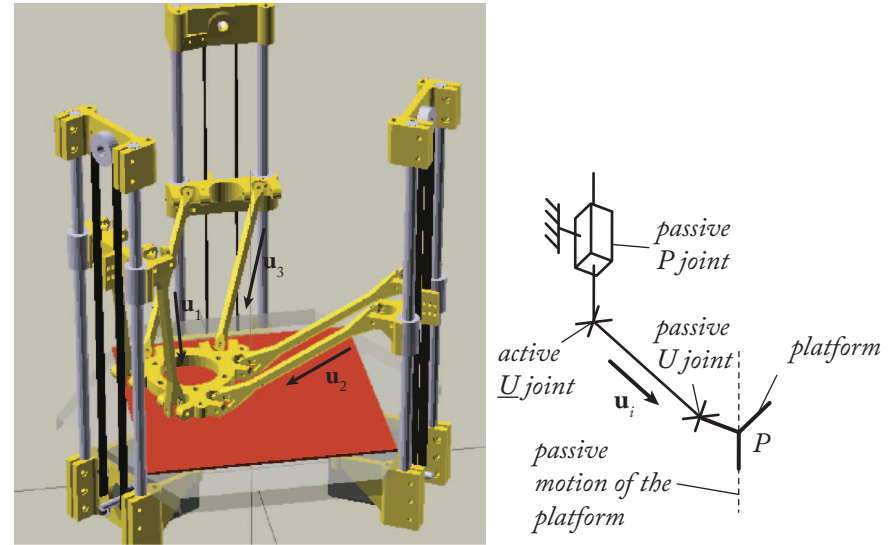

(a) Schematics of the architecture: a 3- (b) Its hidden robot leg: a $P U U$ $\underline{P} U U$ robot with the three actuated $\underline{P}$ joints leg; thus, the hidden robot is a in parallel 3-P $\underline{U U}$ robot with the three passive $P$ joints in parallel leading to an uncontrollable translation along the $P$ joints direction

Fig. 10. The UraneSX robot and its hidden robot leg

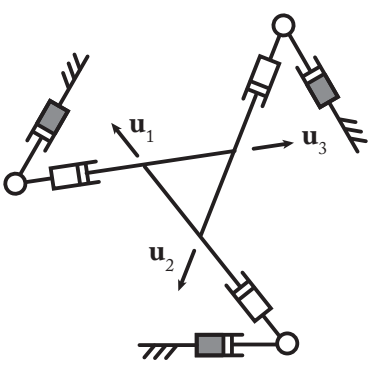

(a) The $3-\underline{P} R P$ robot

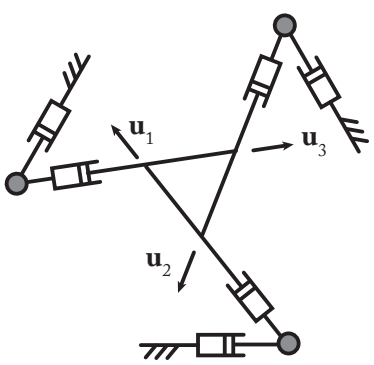

(b) Its hidden robot model: a 3 $P R P$ robot known to be uncontrollable
Fig. 11. The $3-P R P$ robot and its hidden robot model (the grey joints denote the actuated joints)

B. Robots which are partially controllable in their whole workspace using the leg direction observation

The hidden robot model can be used to analyze and understand the singularities of the mapping and to study if a global diffeomorphism exists between the space of the observed element and the Cartesian space. However, not finding a global diffeomorphism does not necessarily mean that the robot is not controllable. This only means that the robot will not be able to access certain zones of its workspace (the zones corresponding to the assembly modes of the hidden robot model which are not contained in the same aspect as the one of the robot initial configuration). This is of course a problem if the operational workspace of the real robot is fully or partially included in these zones.

Robots belonging to this category are probably the most numerous. They are those for which the hidden robot models have several possible assembly modes, whatever is the number of observed leg directions. Presenting an exhaustive list of robots of this category is totally impossible because it requires the analysis of the assembly modes of all hidden robot models for each robot architecture. However, some examples can be provided. 


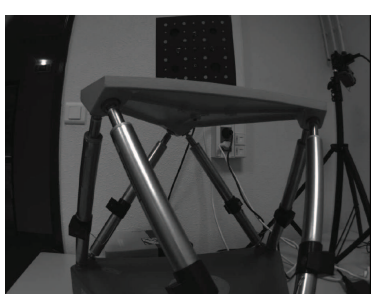

(a) The Gough-Stewart platform from DeltaLab: a $6-U P S$ robot

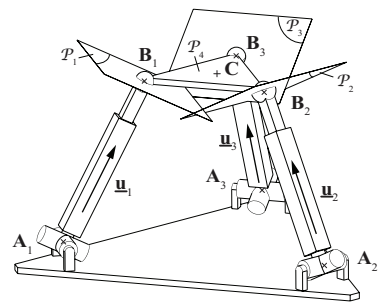

(b) Its hidden robot model: a $3-$ $U P S$ robot (when three legs are $\overline{\text { observed) }}$

Fig. 12. The Gough-Stewart platform and its hidden robot model

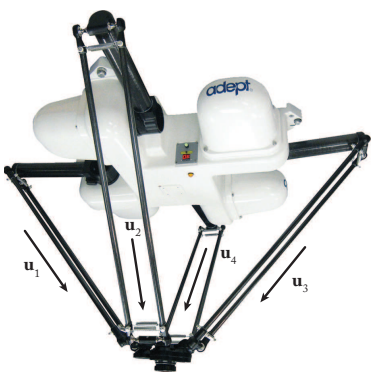

(a) The Adept Quattro: a $4-\underline{R}-$ $2-U S$ robot

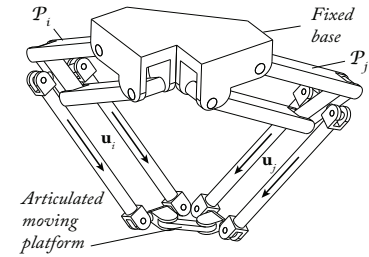

(b) Its hidden robot model: a 2 $\Pi-2-\underline{U U}$ robot (when two legs are observed)
Fig. 13. The Adept Quattro and its hidden robot model

Examples of such types of robots (the Gough-Stewart platform (Fig. 12) and the Adept Quattro (Fig. 13)) have been presented in [19], [21], [22]. More specifically, in [21], [22], it was shown (numerically but also experimentally) that the Adept Quattro [41] controlled through leg direction observation has always at least two assembly modes of the hidden robot model, whatever the number of observed legs. As a result, some areas of the robot workspace were never reachable from the initial configuration. Figure 14 shows a desired robot configuration that was impossible to reach even if all robot legs were observed.

It should be mentioned that, even if it is out of the scope of the present paper, it can be verified if the operational workspace of the real robot is fully or partially included in the aspects of the hidden robot models. This problem may be complex, but can be solved using some advanced tools such as interval analysis [25] or Cylindrical Algebraic Decomposition $(C A D)$ [42]. It should also be mentioned that a Maple library for the $C A D$ has been developed by IRCCyN and is available under request on [43].

C. Robots which are fully controllable in their whole workspace using the leg direction observation

Robots of this category are those for which there exists a global diffeomorphism between the leg direction space and Cartesian space for all workspace configurations. Their hidden robot models have only one possible assembly mode. Once again, presenting an exhaustive list of robots of this category is totally impossible because it requires the analysis of the assembly modes of all hidden robot models for each robot architecture.

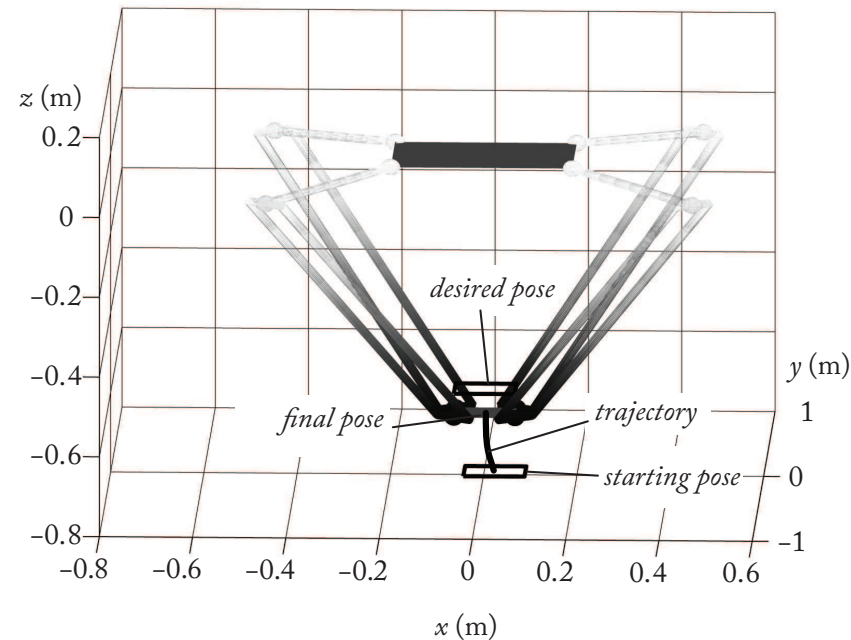

Fig. 14. Desired and final position of the Quattro when all legs are observed.

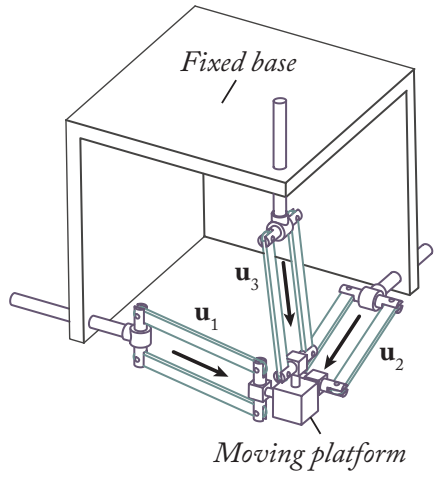

(a) Kinematic chain

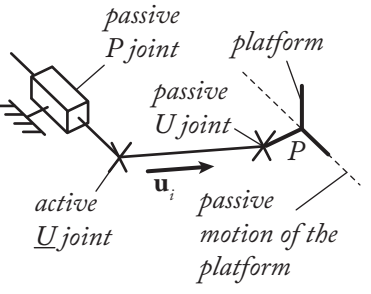

(b) The hidden robot leg
Fig. 15. The Orthoglide and its hidden robot leg.

However, we show here for the first time robots belonging to this category. Let us consider the Orthoglide [44] designed at IRCCyN (Fig. 15(a)). This robot is a mechanism with 3 translational dof of the platform. It is composed of three identical legs made of $\underline{P} R \Pi R$ architecture, or also with $\underline{P} U U$ architecture, the $\underline{P}$ joint of each leg being orthogonal.

Let us consider the second type of leg which is simpler to analyze (even if the following results are also true for the first type of leg). If the link between the two passive $U$ joints is observed, from Section III, the hidden robot leg has a $P \underline{U} U$ architecture with, of course, two degrees of actuation. As a result, for controlling the three dof of the platform, only two legs need to be observed.

For a fixed configuration of the actuated $\underline{U}$ joint, each leg tip has the possibility to freely move on a line directed along the corresponding $P$ joint direction: this line corresponds to the free motion of the platform due to the virtual passive $P$ joint of each leg, when other legs are disconnected (Fig. 15(b)). Then, estimating the robot pose is equivalent to finding the intersection of two lines in space (three lines if the three legs are observed). As a result, in a general manner, the forward kinematic problem $(f k p)$ may have: 


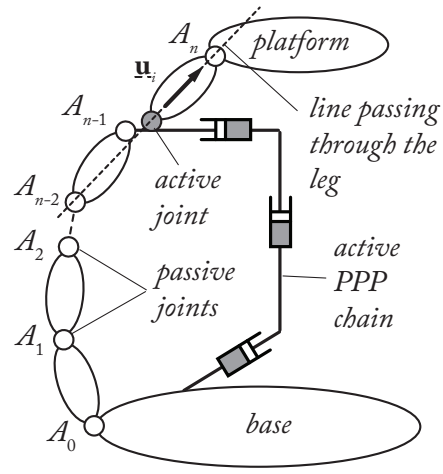

(a) For a general robot

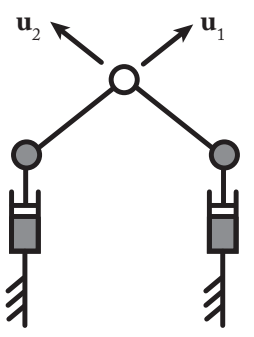

(b) For the $\underline{P R R R} \underline{P}$ : a $\underline{P R} R \underline{R P}$ robot
Fig. 16. The hidden robot leg when the Plücker coordinates of the line passing through the axis of the leg are observed.

- zero solutions (impossible in reality due to the robot geometric constraints),

- an infinity of solutions if and only if the $P$ joints are parallel (not possible for the Orthoglide as all $P$ joints are orthogonal),

- one solution (the only possibility).

Moreover, a simple singularity analysis of all the possible hidden robot models of the Orthoglide could show that they have no Type 2 singularities (which is coherent with the fact that the $f k p$ has only one solution).

By extension of these results, it could be straightforwardly proven that all robots with 3 translational dof of the platform, or with Schönflies motions ( 3 translational dof of the platform plus one rotational dof about one fixed axis), which are composed of identical legs made of $\underline{P} R \Pi R$ architecture, or also with $\underline{P} U U$ architecture and for at least two $P$ joints are not parallel (e.g. the Y-STAR [45]) are fully controllable in their whole workspace using the leg direction observation.

\section{Robots which become fully controllable in their whole workspace if additional information is used}

After this classification, one additional question is to know if, by adding additional information in the controller, the robots which were uncontrollable or partially controllable in their whole workspace can become fully controllable.

For example, it was very recently proven in [46] that, from the projection of the cylindrical leg in the image plane (Fig. 1), it is not only possible to estimate the leg direction, but also the Plücker coordinates of the line passing through the axis of the cylinder, i.e. the direction and location in space of this line. Using this information leads to a modification of the virtual leg as shown in Fig. 16(a): the additional prismatic chain, instead of being passive, becomes active.

This additional information can solve many issues of controllability mentioned above. For example, by estimating the Plücker coordinates of the line passing through its legs, the $\underline{P R R R} \underline{P}$ robot of Section IV-A becomes controllable as the hidden robot model becomes a $\underline{P R} \underline{R P}$ robot (Fig. 16(b)) which is fully controllable.
However, this information may not be enough for some categories of robots, such as for the MePaM [36] for which it was shown in [47] that using the Plücker coordinates of the line passing through the legs leads to a robot which is partially controllable in its whole workspace (eight different assembly modes of the hidden robot model may appear). A similar result could be proven for the GS platform for which the Plücker coordinates do not bring any additional useful information in the controller. For such robots, two main solutions are possible:

- if the robot operational workspace is included in one given aspect of the hidden robot model, the controller may be sufficient to fully control the robot in its operational workspace,

- other features (such as other robot elements (joint locations, other links, etc.)) should be observed to complete the missing information.

Regarding this last point, it is necessary to mention that, in this paper, we only focus on the information that we could extract from the camera, and not from other sensors. Indeed, combining information from different sensors implies some issues of multi-sensor calibration which are not addressed here but that will be part of our future work.

\section{ILLUSTRATIVE EXAMPLES}

\section{A. Case study 1: a 3-PRR planar robot}

1) Presentation of the robot under study: In the present section, we illustrate the present work by analyzing the controllability of a special type of planar $3-\underline{P} R R$ robot with parallel $\underline{P}$ and two coincident platform joints (Fig. 17(a)). In the following of the paper, we consider that:

- $q_{1}, q_{2}$ and $q_{3}$ are the coordinates of the actuators of the real robot,

- the lengths of segments $A_{1} P, A_{2} P$ and $A_{3} P$ are denoted $l_{A_{1} P}, l_{A_{2} P}$ and $l_{A_{3} B}$, respectively, and are equal, i.e. $l=$ $l_{A_{1} P}=l_{A_{2} P}=l_{A_{3} B}$,

- the controlled point on the effector is the point $P$ with coordinates $x$ and $y$ along the $\boldsymbol{x}$ and $\boldsymbol{y}$ axes, respectively,

- the orientation of the platform with respect to the $\boldsymbol{x}$ axis is parametrized by the angle $\phi$,

- the distance between the joints located at points $P$ and $B$ is denoted as $d$.

For this mechanism, Type 1 singularities appear when $\underline{\mathbf{u}}_{i}$ is orthogonal to the direction of the prismatic guide of the leg $i$ (Fig. 17(b)). These singularities represent some workspace boundaries.

For this mechanism, Type 2 singularities appear:

- when $\underline{\mathbf{u}}_{1}$ and $\underline{\mathbf{u}}_{2}$ are collinear (Fig. 18(a)): they appear if and only if the legs 1 and 2 are in antagonistic working modes (' +- ' or ' -+ ', see Fig. 17(b)) for $x=a_{1} / 2$ for any $y$ and $\phi$, i.e. they never appear when the legs 1 and 2 are in working modes ' ++ ' or ' -- ' such as in Fig. 17(a).

- or when $\underline{\mathbf{u}}_{2}$ and $\overrightarrow{P B}$ are collinear (Fig. 18(b)): they may appear for any $x$ and $y$ if and only if the robot reaches constant platform orientations defined by $\cos \phi=$ $a_{2} /(d+l)$ or $\cos \phi=a_{2} /|d-l|$. 

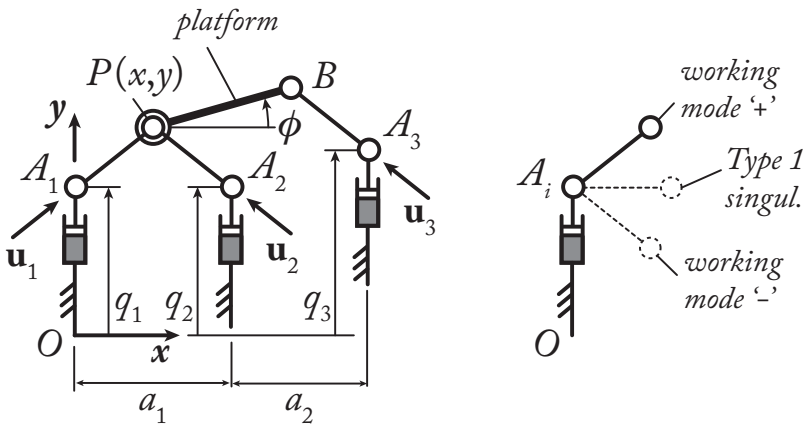

(a) Kinematic architecture of the robot

(b) Kinematic architecture of one robot leg, its Type 1 singularity and its working modes

Fig. 17. Schematics of the $3-\underline{P R R}$ robot.

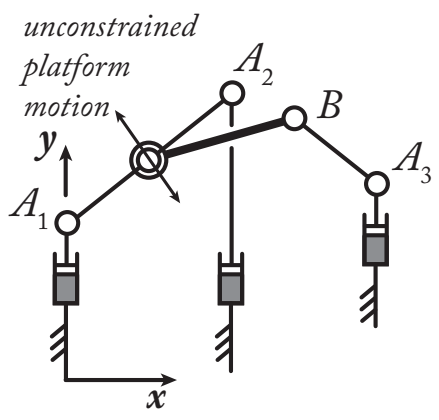

(a) Example of the first case of Type 2 singularity

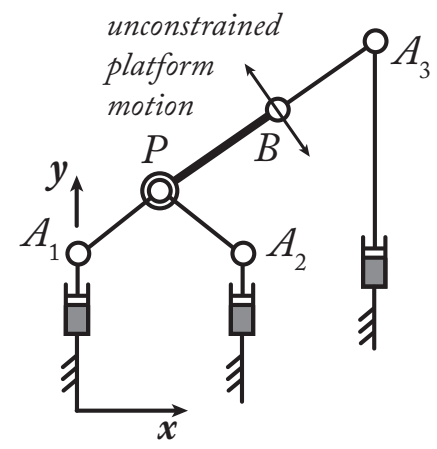

(b) Example of the second case of Type 2 singularity

Fig. 18. Singularities of the $3-\underline{P} R R$ robot.

2) Analysis of the possible hidden robot models: Case 1: Let us now assume that we want to control the $3-\underline{P} R R$ robot depicted at Fig. 17(a) by using the observation of its leg directions $\underline{\mathbf{u}}_{i}$ (see Section II). From Section III, we know that using such a control approach involves the appearance of a hidden robot model. This hidden robot model can be found by straightforwardly using the results of Section III and is a 3-P $\underline{R} R$ robot shown in Fig. 19(a). This robot is known to be architecturally singular (it can freely move along the $\boldsymbol{y}$ axis) and can not be controlled by using only the observation of its leg directions $\underline{\mathbf{u}}_{i}$.

Case 2: As a result, one would logically wonder what should be the necessary information to retain in the controller to servo the robot. By using the results of Section IV-D, we know that, from the projection of the cylindrical leg in the image plane, it is not only possible to estimate the leg direction, but also the Plücker coordinates of the line passing through the axis of the cylinder, i.e. the direction and location in space of this line. Let us consider that we add this information for the estimation of the leg 1 position only. Modifying the hidden robot model according to Fig. 16(a), the corresponding robot model hidden in the controller is depicted in Fig. 19(b): this is a $\underline{P R R}-\{2-P \underline{R} R\}$ robot which is not architecturally singular. In other words, using the Plücker coordinates of the line for leg 1 involves to actuate both the first $P$ and $R$ joints of the corresponding leg, i.e. the virtual leg is a $\underline{P R} R$ leg. For the $\underline{P R R}-\{2-P \underline{R} R\}$ robot, it is possible to prove that two assembly modes exist which are separated by a Type 2 singularity at $\phi=0$ or $\pi$ (for any $x$ and $y$ ). For both assembly modes, the end-effector position is the same, while the orientation is different. Thus, the robot is not fully controllable in its whole workspace.

Case 3: From the result that, using the Plücker coordinates of the line passing through the axis of the cylinder, the leg of the virtual robot becomes a $\underline{P R R}$ leg, it is possible to understand what is the minimal set of information to provide to the controller to fully control the robot in the whole workspace: we need to use the Plücker coordinates of the lines passing through legs 1 and 3 and the direction of the leg 2. In such a case, the hidden robot model is a $P \underline{R R}-\{2-\underline{P R R}\}$ robot depicted in Fig. 19(c). It is possible to prove that this robot has no Type 2 singularity and can freely access its whole workspace.

3) Simulation results: Simulations are performed on an Adams mockup of the $3-\underline{P R R}$ robot with the following values for the geometric parameters: $l=1 \mathrm{~m}, d=0.4 \mathrm{~m}, a_{1}=0.4 \mathrm{~m}$ and $a_{2}=0.25 \mathrm{~m}$. This virtual mockup is connected to Matlab/Simulink via the module Adams/Controls. The controller presented in Section II is applied with a value of $\lambda$ assigned to 20 .

The initial configuration of the robot end-effector is $x_{0}=$ $0.20 \mathrm{~m}, y_{0}=0.98 \mathrm{~m}$ and $\phi_{0}=-45 \mathrm{deg}$. We want to reach the end-effector configuration $x_{f}=0.20 \mathrm{~m}, y_{f}=1.03 \mathrm{~m}$ and $\phi_{f}=-10 \mathrm{deg}$. For that, we use the three possible controllers (Cases 1, 2 and 3) proposed in the previous Section and simulate the robot behavior with the Adams mockup during 1 second. For the three cases, the errors on the used observed features (either the leg directions or the Plücker coordinates of the lines) tends to zero at the end of the simulation. However, this is not necessary the case for the end-effector configuration (Table I).

With the controller of Case 1 based on the observation of the leg directions only, the robot is not able to attain the final end-effector configuration. Moreover, the end-effector position is unchanged (while its orientation has been modified) which is coherent with the results of the previous section: the corresponding hidden robot is architecturally singular and its motion along the $\boldsymbol{y}$ axis is uncontrollable.

For the two other controllers, the convergence towards the desired end-effector pose is achieved. 


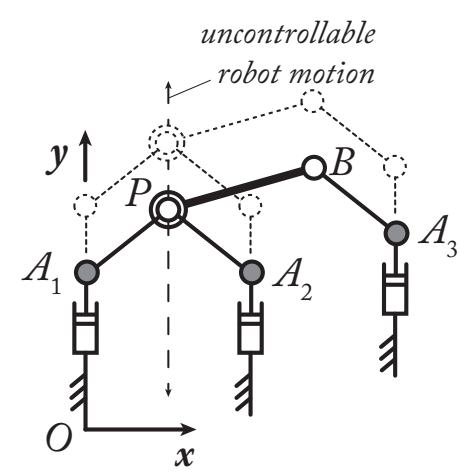

(a) When all leg directions $\underline{\mathbf{u}}_{i}$ are observed (Case 1): a 3-P $\underline{R} R$ robot

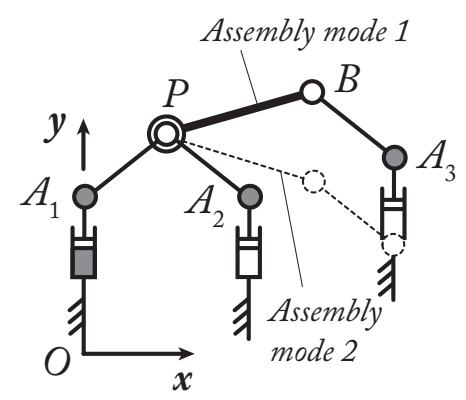

(b) When all leg directions $\underline{\mathbf{u}}_{i}$ and the Plücker coordinates of the line passing through the leg 1 are observed (Case 2): a $\underline{P R R}-\{2-P \underline{R} R\}$ robot

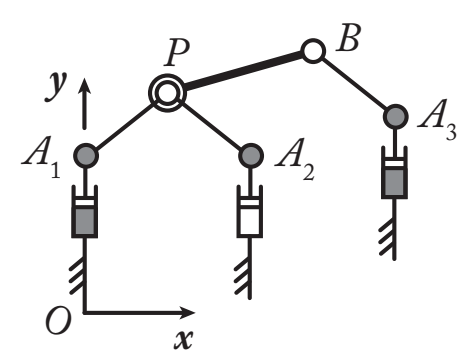

(c) When all leg directions $\underline{\mathbf{u}}_{i}$ and the Plücker coordinates of the lines passing through the legs 1 and 3 are observed (Case 3): a $P \underline{R} R-\{2-\underline{P R R}\}$ robot

Fig. 19. Hidden robots involved in the tested visual servoings of the $3-\underline{P R R}$ robot.

Now, we change the desired end-effector configuration $x_{f}=$ $0.20 \mathrm{~m}, y_{f}=1.03 \mathrm{~m}$ and $\phi_{f}=+10 \mathrm{deg}$. The results for the end-effector convergence are provided in Table II.

With the controller of Case 1, the results are unchanged: the robot is not able to reach the desired configuration.

With the controller of Case 2 based on the observation of the Plücker coordinates of the line passing through the leg 1 and the other leg directions, the robot attains the final end-effector position, but not the correct orientation. This is coherent with the results of the previous section: the corresponding hidden robot has two assembly modes with similar end-effector positions but different orientations. It can
TABLE I

FINAL END-EFFECTOR CONFIGURATION FOR THE DESIRED END-EFFECTOR CONFIGURATION $x_{f}=0.20 \mathrm{M}, y_{f}=1.03 \mathrm{M} \mathrm{AND} \phi_{f}=-10 \mathrm{DEG}$

\begin{tabular}{llll}
\hline \hline & $x(\mathrm{~m})$ & $y(\mathrm{~m})$ & $\phi(\mathrm{deg})$ \\
\hline Case 1 & 0.20 & 0.98 & -10 \\
Case 2 & 0.20 & 1.03 & -10 \\
Case 3 & 0.20 & 1.03 & -10 \\
\hline \hline
\end{tabular}

TABLE II

FINAL END-EFFECTOR CONFIGURATION FOR THE DESIRED END-EFFECTOR CONFIGURATION $x_{f}=0.20 \mathrm{M}, y_{f}=1.03 \mathrm{M} \mathrm{AND} \phi_{f}=+10 \mathrm{DEG}$

\begin{tabular}{llll}
\hline \hline & $x(\mathrm{~m})$ & $y(\mathrm{~m})$ & $\phi(\mathrm{deg})$ \\
\hline Case 1 & 0.20 & 0.98 & -10 \\
Case 2 & 0.20 & 1.03 & -10 \\
Case 3 & 0.20 & 1.03 & +10 \\
\hline \hline
\end{tabular}

be proven that, for the given robot geometric parameters, the two assembly modes of the $\underline{P R R}-\{2-P \underline{R} R\}$ robot for the given observed features at the desired final robot configuration are:

- $x_{1}=0.20 \mathrm{~m}, y_{1}=1.03 \mathrm{~m}$ and $\phi_{1}=+10 \mathrm{deg}$, and

- $x_{2}=0.20 \mathrm{~m}, y_{2}=1.03 \mathrm{~m}$ and $\phi_{2}=-10 \mathrm{deg}$.

Thus, the robot has converged towards the second assembly mode, which was not the desired one. However, this second assembly mode was reached during the first simulation, because it is enclosed in the same workspace aspect corresponding to the initial robot configuration.

Finally, with the controller of Case 3 based on the observation of the Plücker coordinates of the lines passing through the legs 1 and 3 and the leg 2 direction, the robot reached the desired configuration. This result was expected from the previous Section.

\section{B. Case study 2: a 3-PRS spatial robot}

1) Presentation of the robot under study: In the section, we analyze the controllability of a special type of spatial 3-PRS robot with parallel $\underline{P}$ joints which is indeed the kinematic representation of the Sprint Z3 machine from Siemens (Fig. 20). This robot is a zero-torsion robot [38], which means that it has three coupled dof which are usually taken as the translation along $z$ and two rotations. Moreover, by taking into account the Tilt-and-Torsion angle formalism [48], it was demonstrated in [38] that the torsion angle was always zero. As a result, we propose to parameterize here the robot dof as:

- the translation along $z$ of the point $B_{1}$ denoted as $z$,

- the first two angles of the Tilt-and-Torsion parameterization [48], i.e. the azimuth and tilt angles denoted as $\phi$ and $\theta$ respectively.

In the following of the paper, we consider that:

- $q_{1}, q_{2}$ and $q_{3}$ are the coordinates of the actuators of the real robot (positions of points $A_{i}$ along $z$ ),

- due to the PRS architecture of each leg, the points $B_{i}$ (centers of the spherical joints) are constrained to move in a vertical plane denoted as $\mathcal{P}_{i}$ whose normal vector is parallel to the $R$ joint axis (Fig. 20(b)), 


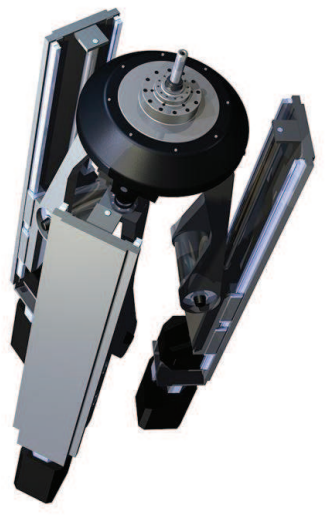

(a) A design known as the Sprint Z3 machine

Fig. 20. The spatial 3-$R S$ robot with parallel $\underline{P}$ joints.

- the relative orientation between $\mathcal{P}_{1}$ and $\mathcal{P}_{2}$ (and $\mathcal{P}_{2}$ and $\mathcal{P}_{3}$ ) is $120 \mathrm{deg}$. (obviously around the vertical axis $z$ ),

- the lengths of segments $A_{1} B_{1}, A_{2} B_{2}$ and $A_{3} B_{3}$ are denoted $l_{A_{1} B_{1}}, l_{A_{2} B_{2}}$ and $l_{A_{3} B_{3}}$, respectively, and are equal, i.e. $l=l_{A_{1} B_{1}}=l_{A_{2} B_{2}}=l_{A_{3} B_{3}}$,

- the prismatic joints are equidistant with a fixed distance $d$ between them,

- the points $B_{1}, B_{2}$ and $B_{3}$ of the platform forms an equilateral triangle of circumcircle with radius $R$.

For this mechanism, Type 1 singularities appear when $\underline{\mathbf{u}}_{i}$ is orthogonal to the direction of the prismatic guide of the leg $i$ [38]. These singularities represent some workspace boundaries. Type 2 singularities are more complex and are studied in [49].

2) Analysis of the possible hidden robot models: Case 1: Let us now assume that we want to control the $3-\underline{P} R S$ robot depicted at Fig. 20 by using the observation of its leg directions $\underline{\mathbf{u}}_{i}$ (see Section II). From Section III, we know that using such a control approach involves the appearance of a hidden robot model. This hidden robot model can be found by straightforwardly using the results of Section III and is a 3-P $\underline{R} S$ robot shown in Fig. 21(a). This robot is known to be architecturally singular (it can freely move along the $z$ axis) and can not be controlled by using only the observation of its leg directions $\underline{\mathbf{u}}_{i}$.

Case 2: As a result, one would logically wonder what should be the necessary information to retain in the controller to servo the robot. For instance, let us use the Plücker coordinates of the line passing through the axis of the cylinder (see Section IV-D), i.e. the direction and location in space of this line. Let us consider that we add this information for the estimation of the legs 1 and 2 positions. Modifying the hidden robot model according to Fig. 16(a), the corresponding robot model hidden in the controller is depicted in Fig. 21(b): this is a $\{2-\underline{P R} S\}-P \underline{R} S$ robot which is not architecturally singular. In other words, using the Plücker coordinates of the line for legs 1 and 2 involves to actuate both the first $P$ and $R$ joints of the corresponding legs, i.e. the virtual legs are $\underline{P R} S$ legs. For the $\{2-\underline{P R S}\}-P \underline{R} S$ robot, it is possible to prove that two assembly
TABLE III

FINAL END-EFFECTOR CONFIGURATION FOR THE DESIRED END-EFFECTOR CONFIGURATION $z_{f}=0.40 \mathrm{M}, \phi_{f}=-90$ DEG AND $\theta_{f}=+10$ DEG

\begin{tabular}{llll}
\hline \hline & $z(\mathrm{~m})$ & $\phi(\mathrm{deg})$ & $\theta(\mathrm{deg})$ \\
\hline Case 1 & 0.20 & -90 & -10 \\
Case 2 & 0.40 & -90 & -10 \\
Case 3 & 0.40 & -90 & +10 \\
\hline \hline
\end{tabular}

modes exist. Indeed, for this robot, when fixing the position of points $B_{1}$ and $B_{2}$ (which is the case when actuating the $P$ and $R$ joints of the legs 1 and 2), the platform can freely rotate around $\left(B_{1} B_{2}\right)$. Thus, $B_{3}$ performs a circle which will intersect with the line corresponding of the free motion of the leg 3 tip when the platform is disconnected and the $R$ joint is actuated only. As a result, the maximal number of solutions of the $f k p$ is equal to two. For both assembly modes, the endeffector position is the same, while the orientation is different. Thus, the robot is not fully controllable in its whole workspace. Case 3: From the result that, using the Plücker coordinates of the line passing through the axis of the cylinder, the leg of the virtual robot becomes a $\underline{P R S}$ leg, it is possible to understand what is the minimal set of information to provide to the controller to fully control the robot in the whole workspace: we need to use all the Plücker coordinates of the lines passing through legs 1 to 3 . In such a case, the hidden robot model is a 3- $\underline{P R S}$ robot depicted in Fig. 21(c). It is possible to prove that this robot has no Type 2 singularity and can freely access to its whole workspace.

3) Simulation results: Simulations are performed on an Adams mockup of the $3-\underline{P R S}$ robot with the following values for the geometric parameters: $l=0.5 \mathrm{~m}, d=0.4 \mathrm{~m}, R=$ $0.1 \mathrm{~m}$. This virtual mockup is connected to Matlab/Simulink via the module Adams/Controls. The controller presented in Section II is applied with a value of $\lambda$ assigned to 20 .

The initial configuration of the robot end-effector is $z_{0}=$ $0.20 \mathrm{~m}, \phi_{0}=-90 \mathrm{deg}$ and $\theta_{0}=-10 \mathrm{deg}$. We want to reach the end-effector configuration $z_{f}=0.40 \mathrm{~m}, \phi_{f}=-90 \mathrm{deg}$ and $\theta_{f}=+10 \mathrm{deg}$. For that, we use the three possible controllers (Cases 1, 2 and 3) proposed in the previous Section and simulate the robot behavior with the Adams mockup during 1 second. For the three cases, the errors on the used observed features (either the leg directions or the Plücker coordinates of the lines) tends to zero at the end of the simulation. However, this is not necessary the case for the end-effector configuration (Table III).

With the controller of Case 1 based on the observation of the leg directions only, the robot is not able to attain the final end-effector configuration. Moreover, the end-effector position is unchanged which is coherent with the results of the previous section: the corresponding hidden robot is architecturally singular and its motion along the $z$ axis is uncontrollable.

With the controller of Case 2 based on the observation of the Plücker coordinates of the line passing through the legs 1 and 2 and the other leg direction, the robot attains the final end-effector position, but not the correct orientation. 


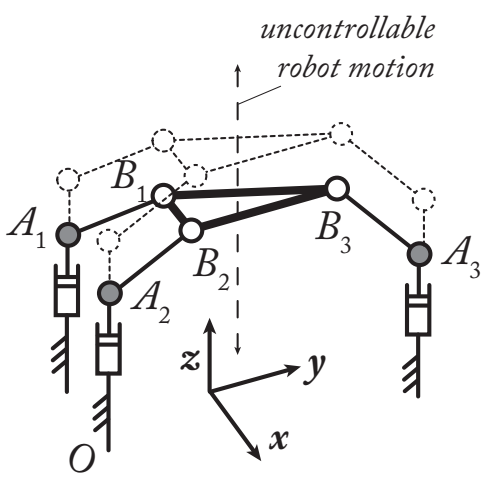

(a) When all leg directions $\underline{\mathbf{u}}_{i}$ are observed (Case 1): a 3-P $\underline{R} S$ robot

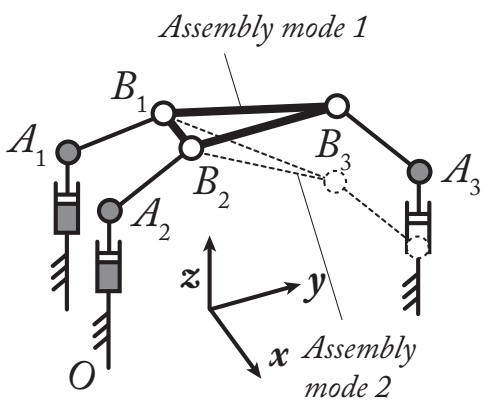

(b) When all leg directions $\underline{\mathbf{u}}_{i}$ and the Plücker coordinates of the line passing through the legs 1 and 2 are observed (Case 2): a $\{2-P R S\}-P R S$ robot

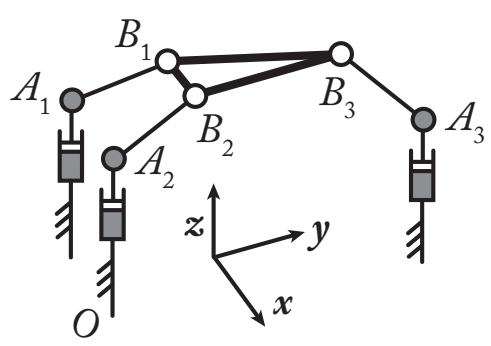

(c) When all leg directions $\underline{\mathbf{u}}_{i}$ and the Plücker coordinates of the lines passing through the legs 1 and 3 are observed (Case 3): a 3-PRS robot

Fig. 21. Hidden robots involved in the tested visual servoings of the 3-PRS robot (projection in the $y z$ plane $-R$ and $S$ joints at $A_{i}$ and $B_{i}$, respectively, are drawn with the same symbol for the sake of clarity of the drawing).

This is coherent with the results of the previous section: the corresponding hidden robot has two assembly modes with similar end-effector positions but different orientations. It can be proven that, for the given robot geometric parameters, the two assembly modes of the $\{2-\underline{P R S}\}-P \underline{R} S$ robot for the given observed features at the desired final robot configuration are:

- $z_{f}=0.40 \mathrm{~m}, \phi_{f}=-90 \mathrm{deg}$ and $\theta_{f}=-10 \mathrm{deg}$, and

- $z_{f}=0.40 \mathrm{~m}, \phi_{f}=-90 \mathrm{deg}$ and $\theta_{f}=+10 \mathrm{deg}$.

Thus, the robot has converged towards the second assembly mode, which was not the desired one. However, this second assembly mode was reached during the first simulation, because it is enclosed in the same workspace aspect corresponding to the initial robot configuration.

Finally, with the controller of Case 3 based on the observation of the Plücker coordinates of the lines passing through the legs 1 to 3 , the robot reached the desired configuration. This result was expected from the previous Section.

\section{Discussion}

The results from the simulations show the real added value of the hidden robot concept. The hidden robot being a tangible visualization of the mapping between the observation space and the real robot Cartesian space, it is possible:

- to prove if the studied robot is controllable or not in its whole workspace by the use of quite simple mechanism analysis tools,

- to understand the features to observe to ensure the controllability of the robot in its whole workspace.

To conclude this part, it is necessary to mention that:

- in our simulations, we have considered that the observed features were not noisy, which is not true in reality. This has been simply assumed for two main reasons: (i) robustness of these types of controllers was already shown in previous works (e.g. [12], [14], [22]) and (ii) adding noise would have made the analysis of the convergence results in the controllers of Case 1 and 2 more difficult to explain, without bringing any added value to these simulations.

- the results for the controller of Case 3 for the first case study would have been the same if the Plücker coordinates of the line 2 were observed instead of those of the line 1 . The choice of the best leg to observe could have been done by a procedure presented in [19] which ensures to select the legs that lead to the best endeffector accuracy. However, this was out of the scope of the present paper.

- in the whole paper, it is considered that the sensor measurement space is the same as the leg direction space. However, for example using a camera, the leg directions are not directly measured but rebuilt from the observation of the legs limbs projection in the 2D camera space [12]. Thus, for the leg reconstruction, the mapping between the camera space and the real 3D space is involved, and it is not free of singularities (see [50] for an example of mapping singularities). In the neighborhood of mapping singularities, the robot accuracy will also tend to decrease. As a result, this mapping should be considered in the accuracy computation and in the selection of the legs to observe.

\section{CONCLUSIONS}

This paper has presented a tool named the "hidden robot concept" that is well addressed for analyzing the controllability of parallel robots in leg-observation-based visual servoing techniques. It was shown that the mentioned visual servoing 
techniques involved the existence of a virtual robot model, hidden into the controller, that was different from the real controlled robot. Considering this hidden robot model allowed a minimal representation to be found for the leg-observationbased control of the studied robots that is linked to a virtual hidden robot which is a tangible visualization of the mapping between the observation space and the real robot Cartesian space. It was shown that the hidden robot model could be used to:

1) explain why the observed robot which is composed of $n$ legs can be controlled using the observation of only $m$ leg directions $(m<n)$, knowing the fact that the minimal number of observed legs should be, for 3D unit vectors, an integer greater than $n / 2$,

2) prove that there does not always exist a global diffeomorphism between the Cartesian space and the leg direction space,

3) simplify the singularity analysis of the mapping between the leg direction space and the Cartesian space by reducing the problem to the singularity analysis of a new robot,

4) certify that the robot will not converge to local minima, through the application of tools developed for the singularity analysis of robots.

A general way to find the hidden robot models corresponding to the real robot controlled via leg-observation-based visual servoing techniques was shown and the hidden robot models of some well known classes of parallel robots were studied. It was proven that, using this concept, it is possible to demonstrate, using tools developed by the mechanical design community, that the robot could be controlled or not with the aforementioned visual servoing techniques. Based on these results, a classification into families of robots which are not controllable, partially or fully controllable in their whole workspace using the aforementioned servoing technique was proposed. Moreover, insights about the features that should be additionally observed to ensure that the robots could be fully controllable in their whole workspace were discussed.

Finally, numerical simulations made on Adams mockup of a planar robot demonstrated the validity of the theoretical developments.

Thus, the concept of the hidden robot model, associated with mathematical tools developed by the mechanical design community, is a powerful tool able to analyze the intrinsic properties of some controllers developed by the visual servoing community. Moreover, this concept showed that in some visual servoing approaches, stacking several interaction matrices to derive a control scheme without doing a deep analysis of the intrinsic properties of the controller is clearly not enough. Further investigations are required.

\section{ACKNOWLEDGEMENTS}

This work was supported by the French ANR project ARROW (ANR-2011BS3-006-01) and the EU project Feder RobotEx.

\section{REFERENCES}

[1] J. Merlet, "www-sop.inria.fr/members/jean-pierre.merlet/merlet.html," 2012. [Online]. Available: www-sop.inria.fr/members/JeanPierre.Merlet/merlet.html

[2] D. Dementhon and L. Davis, "Model-based object pose in 25 lines of codes," International Journal of Computer Vision, vol. 15, pp. 123-141, 1995.

[3] M. Dhome, M. Richetin, J. Lapresté, and G. Rives, "Determination of the attitude of 3-d objects from a single perspective view," IEEE Transactions on Pattern Analysis and Machine Intelligence, vol. 11, no. 12 , pp. $1265-1278,1989$.

[4] D. Lowe, "Three-dimensional object recognition from single twodimensional images," Artificial Intelligence, vol. 31, pp. 355-394, 1987.

[5] R. Horaud, F. Dornaika, and B. Espiau, "Visually guided object grasping," IEEE Transactions on Robotics and Automation, vol. 14, no. 4, pp. 525-532, 1998.

[6] P. Martinet, J. Gallice, and D. Khadraoui, "Vision based control law using 3D visual features," in Proceedings of the World Automation Congress, WAC96, Robotics and Manufacturing Systems, vol. 3, Montpellier, France, May 1996, pp. 497-502.

[7] E. Marchand and F. Chaumette, "Virtual visual servoing: A framework for real-time augmented reality," Computer Graphics Forum, vol. 21, no. 3, pp. 289-298, 2002.

[8] A. Traslosheros, J. Sebastian, L. Angel, F. Roberti, and R. Carelliz, "Visual servoing of a parallel robot system," in IEEE International Symposium on Intelligent Signal Processing, 2007.

[9] Z. Qi and J. McInroy, "Improved image based visual servoing with parallel robot," Journal of Intelligent Robot Systems, vol. 53, pp. 359379, 2008.

[10] R. Garrido, A. Soria, and M. Trujano, "Visual pid control of a redundant parallel robot," in 5th International Conference on Electrical Engineering, Computing Science and Automatic Control (CCE 2008), 2008.

[11] M. Trujano, R. Garrido, and A. Soria, "Robust visual control of parallel robots under uncertain camera orientation," International Journal of Advanced Robotic Systems, vol. 9, 2012.

[12] N. Andreff, A. Marchadier, and P. Martinet, "Vision-based control of a Gough-Stewart parallel mechanism using legs observation," in Proceedings of the IEEE International Conference on Robotics and Automation, ICRA'05, Barcelona, Spain, April 18-22 2005, pp. 25462551.

[13] V. Gough and S. Whitehall, "Universal tyre test machine," in Proceedings of the FISITA 9th International Technical Congress, May 1962, pp. 117-317.

[14] N. Andreff, T. Dallej, and P. Martinet, "Image-based visual servoing of gough-stewart parallel manipulators using legs observation," International Journal of Robotics Research, vol. 26, no. 7, pp. 677-687, 2007.

[15] E. Ozgur, N. Andreff, and P. Martinet, "Dynamic control of the quattro robot by the leg edgels," in Proceedings of the IEEE International Conference on Robotics and Automation, ICRA11, Shanghai, China, May 9-13 2011.

[16] N. Andreff and P. Martinet, "Vision-based kinematic modelling of some parallel manipulators for control purposes," in Proceedings of EuCoMeS, the First European Conference on Mechanism Science, Obergurgl, Austria, 2006.

[17] B. Espiau, F. Chaumette, and P. Rives, "A new approach to visual servoing in robotics," IEEE Transactions on Robotics and Automation, vol. 8, no. 3, 1992.

[18] F. Chaumette, The Confluence of Vision and Control, ser. LNCIS. Springer-Verlag, 1998, no. 237, ch. Potential problems of stability and convergence in image-based and position-based visual servoing, pp. 6678.

[19] S. Briot and P. Martinet, "Minimal representation for the control of Gough-Stewart platforms via leg observation considering a hidden robot model," in Proceedings of the 2013 IEEE International Conference on Robotics and Automation (ICRA 2013), Karlsruhe, Germany, May, 6-10 2013.

[20] S. Caro, W. Khan, D. Pasini, and J. Angeles, "The rule-based conceptual design of the architecture of serial schonflies-motion generators," Mechanism and Machine Theory, vol. 45, no. 2, pp. 251-260, 2010.

[21] V. Rosenzveig, S. Briot, and P. Martinet, "Minimal representation for the control of the Adept Quattro with rigid platform via leg observation considering a hidden robot model," in Proceedings of the IEEE/RSJ International Conference on Intelligent Robots and Systems (IROS 2013), Tokyo Big Sight, Japan, 2013. 
[22] V. Rosenzveig, S. Briot, P. Martinet, E. Ozgur, and N. Bouton, "A method for simplifying the analysis of leg-based visual servoing of parallel robots," in Proc. 2014 IEEE Int. Conf. on Robotics and Automation (ICRA 2014), Hong Kong, China, May 2014.

[23] N. Andreff, B. Espiau, and R. Horaud, "Visual servoing from lines," International Journal of Robotics Research, vol. 21, no. 8, pp. 679700, 2002.

[24] J. Plücker, "On a new geometry of space," Philosophical Transactions of the Royal Society of London, vol. 155, pp. 725-791, 1865.

[25] J. Merlet, Parallel Robots, 2nd ed. Springer, 2006

[26] F. Chaumette and S. Huchinson, Handbook of robotics. Springer, 2008, ch. 24: Visual Servoing and Visual Tracking.

[27] F. Chaumette and S. Hutchinson, "Visual servo control part i: Basic approaches," IEEE Robotics and Automation Magazine, vol. 13, no. 4, pp. 82-90, 2006.

[28] —, "Visual servo control, part ii: Advanced approaches," IEEE Robotics and Automation Magazine, vol. 14, no. 1, pp. 109-118, 2007.

[29] M. Carricato and V. Parenti-Castelli, "Singularity-free fully-isotropic translational parallel manipulators," International Journal of Robotics Research, vol. 21, no. 2, pp. 161-174, 2002.

[30] X. Kong and C. Gosselin, "A class of 3-dof translational parallel manipulators with linear input-output equations," in Proceedings of the Workshop on Fundamental Issues and Future Research Directions for Parallel Mechanisms and Manipulators, Québec City, QC, Canada, October 2002, pp. 3-4.

[31] G. Gogu, "Structural synthesis of fully-isotropic translational parallel robots via theory of linear transformations," European Journal of Mechanics. A/Solids, vol. 23, no. 6, pp. 1021-1039, 2004.

[32] C. Gosselin and J. Angeles, "Singularity analysis of closed-loop kinematic chains," IEEE Transactions on Robotics and Automation, vol. 6, no. 3, pp. 281-290, 1990.

[33] D. Zlatanov, I. Bonev, and C. Gosselin, "Constraint singularities of parallel mechanisms," in Proceedings of the IEEE International Conference on Robotics and Automation (ICRA 2002), May 2002.

[34] I. Bonev, D. Zlatanov, and C. Gosselin, "Singularity analysis of 3dof planar parallel mechanisms via screw theory," ASME Journal of Mechanical Design, vol. 125, no. 3, pp. 573-581, 2003.

[35] P. Ben-Horin and M. Shoham, "Singularity analysis of a class of parallel robots based on grassmanncayley algebra," Mechanism and Machine Theory, vol. 41, no. 8, pp. 958-970, August 2006

[36] S. Caro, G. Moroz, T. Gayral, D. Chablat, and C. Chen, "Singularity analysis of a six-dof parallel manipulator using grassmann-cayley algebra and grobner bases," in Proceedings of the Symposium on Brain, Body and Machine, Montreal, QC, Canada, November 10-12 2010.

[37] I. Bonev, "Geometric analysis of parallel mechanisms," Ph.D. dissertation, Université Laval, QC, Canada, nov 2002

[38] _ "Direct kinematics of zero-torsion parallel mechanisms," in Proceedings of the 2008 IEEE International Conference on Robotics and Automation (ICRA 2008), 2008.

[39] S. Krut, O. Company, M. Benoit, H. Ota, and F. Pierrot, "I4: A new parallel mechanism for Scara motions," in Proceedings of the 2003 International Conference on Robotics and Automation (ICRA 2013), 2003.

[40] O. Company and F. Pierrot, "Modelling and preliminary design issues of a 3-axis parallel machine-tool," Mechanisms and Machine Theory, vol. 37, pp. 1325-1345, 2002.

[41] V. Nabat, M. de la O Rodriguez, O. Company, S. Krut, and F. Pierrot, "Par4: very high speed parallel robot for pick-and-place," in Proceedings of the 2005 IEEE/RSJ International Conference on Intelligent Robots and Systems (IROS 2005)., 2005.

[42] D. Chablat, G. Moroz, and P. Wenger, "Uniqueness domains and non singular assembly mode changing trajectories," in Proceedings of the 2011 IEEE International Conference on Robotics and Automation (ICRA 2011), Shanghai, China, 2011.

[43] SiRoPa Toolbox. [Online]. Available: http://www.irccyn.ecnantes.fr/ chablat/SIROPA

[44] D. Chablat and P. Wenger, "Architecture optimization of a 3-dof parallel mechanism for machining applications, the Orthoglide," IEEE Transactions on Robotics and Automation, vol. 19, no. 3, pp. 403-410, 2003.

[45] J. Hervé, "Group mathematics and parallel link mechanisms," in Proceedings of the IMACS/SICE International Symposium. on Robotics, Mechatronics, and Manufacturing Systems, Kobe, Japan, sep 1992, pp. 459-464.

[46] A. Vignolo, S. Briot, P. Martinet, and C. Chen, "Comparative analysis of two types of leg-observation-based visual servoing approaches for the control of the five-bar mechanism," in Proceedings of the 2014
Australasian Conference on Robotics and Automation (ACRA 2014), University of Melbourne, Australia, dec 2014.

[47] A. Vignolo, "Visual servoing of the Monash epicyclic-parallel manipulator," Master's thesis, École Centrale de Nantes, 2014.

[48] S. Briot and W. Khalil, Dynamics of Parallel Robots - From Rigid Links to Flexible Elements. Springer, 2015, iSBN: 978-3-319-19787-6.

[49] S. Briot and I. Bonev, "Singularity loci of zero-torsion parallel mechanisms," in Proceedings of the IEEE/RJS 11th International Conference on Intelligent Robots and Systems (IROS 2008), Nice, France, sep 2008.

[50] H. Michel and P. Rives, "Singularities in the determination of the situation of a robot effector from the perspective view of 3 points," INRIA, Tech. Rep., 1993.

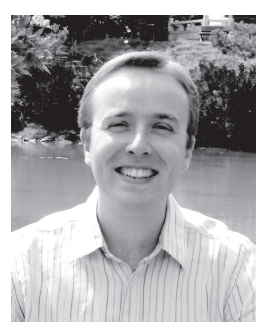

Sébastien Briot received the B.S. and M.S. degrees in Mechanical Engineering in 2004 from the National Institute of Applied Sciences (INSA) of Rennes (France). Then, he began a PhD thesis, supervised by Prof. Vigen Arakelian, at the INSA of Rennes and received the $\mathrm{PhD}$ degree in 2007.

He worked at the École de Technologie Supérieure of Montreal (Canada) with Prof. Ilian Bonev as a postdoctorate fellow in 2008. Since 2009, he is a full-time CNRS researcher at the IRCCyN in Nantes (France) in the Robotics team. Its research fields concern the design optimization of robots and the analysis of their dynamic performance. He also studies the impact of sensor-based controllers on the robot performance. He is the author of more than 30 referred journal papers and 2 inventions.

Dr. Briot received the Award of the Best Ph.D. Thesis in Robotics from the French CNRS for year 2007. In 2011, he received two other awards: the Award for the Best Young Researcher from the French Region Bretagne and the Award for the Best Young Researcher from the French Section of the American Society of Mechanical Engineering (SF-ASME).

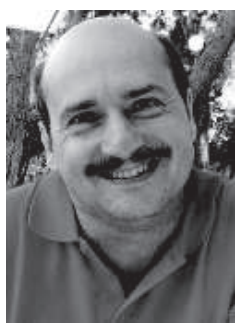

Philippe Martinet received his PhD degree in 1985 from the Blaise Pascal University, France. From 1990 til 2000, he was assistant Professor with CUST in the Electrical Engineering Department, ClermontFerrand. From 2000 until 2011, he was Professor at the Institut Franais de Mécanique Avancée (IFMA), Clermont-Ferrand. He was performing research at the Robotics and Vision Group of LASMEA-CNRS, Clermont-Ferrand. In 2006, he spent one year as a visiting professor in ISRC at the Sungkyunkwan university in Suwon, South Korea.

He was the leader of the group GRAVIR (over 74 person) from 2001 til 2006. From 1997 until 2011, he led the Robotic and Autonomous Complex System team (over 20 persons). From 2008 until 2011, he co-leads a Joint Unit of Technology called "Robotization in meat Indutry", and the Korea France Joint Research Center on Cognitive Personal Transport Service Robot in Suwon (South Korea).

Since 2011, he is a professor at IRCCyN and is the head of the ERASMUS MUNDUS Master program EMARO+. His activities span robot visual servoing, control of autonomous guided vehicles and the modelling / identification / control of redundant / parallel robot. He is the author of more than 280 papers. 


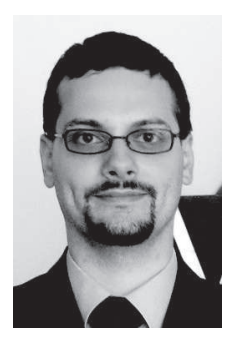

Victor Rosenzveig received the M.S. degree from the EMARO ERASMUS MUNDUS Master program in 2013. He then started a $\mathrm{PhD}$ thesis under the supervision of the Prof. Philippe Martinet and Dr. Sébastien Briot. His research interests span the analysis of sensor-based controllers and the controlbased design of robots. 